\title{
IMPACTS OF PUT OPTION CONTRACT AND SUPPLY CHAIN STRUCTURE IN A MULTI-PERIOD SUPPLY CHAIN WITH UNCERTAIN DEMAND
}

\author{
NANA WAN $^{1, *}$ AND JIANCHANG FAN ${ }^{2}$
}

\begin{abstract}
This paper builds the multi-period optimization models that incorporate put option contract and two supply chain structures to determine the production decision for a supplier and the ordering decision for a manufacturer in a two-stage supply chain. This paper applies the method of dynamic programming to derive the structures of optimal policies and provides an approximate algorithm to evaluate the myopic policies. This paper also conducts numerical examples to illustrate the impacts of put option contract, supply chain structure and demand risk on the members' decisions and total profits as well as the channel's total profit. The results indicate that put option contract can motivate to increase the channel's service level and reduce the manufacturer's inventory risk under two supply chain structures, when compared to the case without put option contract. In the manufacturer-led structure, the channel always benefits from put option contract, the supplier benefits from put option contract with a high option price and a low exercise price, while the manufacturer benefits from put option contract with a low option price and a high exercise price. In the supplier-led structure, the channel and the manufacturer always benefit from put option contract, while the supplier benefits from put option contract with a high option price and a low exercise price. With put option contract, the supplier is more profitable in the manufacturer-led structure than in the supplier-led structure, while the manufacturer and the channel are more profitable in the supplier-led structure than in the manufacturer-led structure. Without and with put option contract, the optimal total profits of two members and the channel will first decrease and then increase in the demand risk. Finally, this paper identifies the explicit conditions under which the multi-period supply chain can be coordinated via put option contract under two supply chain structures. With a coordinating contract, the supplier and the manufacturer are better off compared to the case without put option contract.
\end{abstract}

Mathematics Subject Classification. 90B06, 90C39.

Received February 10, 2020. Accepted June 24, 2020.

\section{INTRODUCTION}

Purchasing and supply management plays a remarkable role in supply chain systems because it affects the efficiency of the members and the channel. Due to the publication of pioneering papers, the purchasing and supply problem has been well studied in a single-period setting via the so-called newsboy model and has

Keywords. Multi-period supply chain, supply chain coordination, put option contract, supply chain structure.

1 School of Economics and Management, Southwest University of Science and Technology, Mianyang 621010, P.R. China.

2 School of Marketing and Logistics Management, Nanjing University of Finance \& Economics, Nanjing 210023, P.R. China.

* Corresponding author: wannana850417@163.com 
been widely extended in the literature via various ways. However, a multi-period structure often underlies the purchasing and supply problem in many cases. This is well known for durable products, but even for perishable products. For example, Hotai Motor Co. Ltd., the distributor of motor vehicles in Taiwan, weekly orders thousands of auto parts from Toyota Motor Company in Japan. It is an effective way to reduce the costs of counting inventory and aggregating transactions as well as save the costs of procurement and transportation [7]. As the purchasing and supply stage is divided into multiple periods, the decision makers are forced to consider the correlation of two successive decisions so that the decision-making process becomes more sophisticated. In this context, the multi-period purchasing and supply problem is worthy to be investigated.

Hedging risk is an intractable problem for supply chain systems that aims to coordinate the purchasing and the supply. It is all known that wholesale price contract cannot incentive the members across the supply chain to work as nearly or exactly the same as a unified system. Instead, it worsens the double marginalization effect and results in supply chain inefficiency. Due to the flexibility and risk-sharing attributes, put option contract, originated in a financial derivative, is introduced as an incentive contract in supply chains to be adopted together with wholesale price contract. A put option gives the buyer of put options the right of cancelling an order of a certain quantity by an expiry date and provides the supplier of put options an option premium to plan capacity in advance [2]. In practice, put option contract has been widely applied in many industries such as computer, agriculture and manufacturing. For example, put options are used to buffer against the negative impact of the unexpected adverse weather conditions, which results in a $\$ 45$ billion in transactions per year in the Chicago Mercantile Exchange in USA [27]. This story stimulates the interest of many researchers to study the impact of put option contract.

This paper considers the interaction between one supplier and one manufacturer within the framework of one two-stage supply chain. Most of previous studies have mainly focused on the supplier-led supply chain in which the supplier acts as the leader of the channel and has the right to design the contract. As the emergence of the large-scale manufacturing enterprise, the manufacturers require more concessions, such as cost reduction, quality promotion and so on, from the suppliers. For example, Boeing and Airbus require their suppliers to reduce the supply costs and improve the qualitative performance to hold their prominence in the aircraft industry. In such case, the manufacturer plays as the leader of the channel and has the right to design the contract. Such two supply chain structures have a different impact on the members' decisions and total profits as well as the channel's total profit. This paper aims to analyze the coordination of the manufacturer-led supply chain and the supplier-led supply chain with put option contract in a multi-period setting. More precisely this paper deals with the following key questions.

(1) With put option contract, how to make the multi-period decisions for two members to optimize their total profits under two supply chain structures?

(2) How does put option contract affect the members' decisions and total profits as well as the channel's total profit?

(3) What impact does the supply chain structure have on the members' decisions and total profits as well as the channel's total profit?

(4) How does the demand risk affect the members' decisions and total profits as well as the channel's total profit?

(5) How to coordinate the multi-period supply chain through put option contract under two supply chain structures?

To answer these above questions, this paper builds the multi-period production models for the supplier and the multi-period ordering models for the manufacturer under put option contract and two supply chain structures. By applying the method of dynamic programming this paper characterizes the structures for optimal policies in two cases. This paper provides an approximate algorithm to evaluate the myopic policies. By taking the model without put option contract as the evaluation criterion, this paper investigates the impact of put option contract on the members' decisions and total profits as well as the channel's total profit. By comparing the model with the manufacturer-led structure against that with the supplier-led structure, this paper examines the impact 
of supply chain structure on the members' decisions and total profits as well as the channel's total profit. By analyzing the impact of the standard deviation of demand, this paper examines the impact of demand risk on the members' decisions and total profits as well as the channel's total profit. On this basis, this paper studies the coordination of the multi-period supply chain under put option contract and two supply chain structures.

This paper makes two main contributions as follows. (1) Previous studies on put option contract have only focused on the supplier-led supply chain in a single-period setting [2,12,23]. This paper distinguishes from the previous literature by incorporating two supply chain structures. Besides that, this paper extends the previous literature by looking at the multi-period setting. To the best of authors' knowledge, this paper is the first to develop the multi-period optimization models with put option contract under two supply chain structures. (2) This paper conducts groups of comparisons to derive meaningful findings with respective to the impacts of put option contract and supply chain structure on the members' decisions and total profits as well as the channel's total profit. This complements to the existing research that only highlights the impact of put option contract on the supplier-led supply chain, but never considers the impact of put option contract on the manufacturer-led supply chain and even the impact of supply chain structure on the supply chain. An all-around research system has been developed in our paper.

The rest of this paper is organized as follows. Section 2 presents a brief literature review. Section 3 details the model description and assumptions. Section 4 analyzes the benchmark model without put option contract. Section 5 analyzes two models with put option contract under two supply chain structures. Section 6 investigates the impacts of put option contract, supply chain structure and demand risk on the members' decisions and total profits as well as the channel's total profit. Section 7 analyzes the coordination of the multi-period supply chain under put option contract and two supply structures. Section 8 concludes the main results and proposes future directions. All the proofs are provided in the Appendix A.

\section{Literature REVIEW}

This paper combines the following research streams together: (1) supply chain management under demand uncertainty, (2) risk hedging with put option contract and (3) the impact of supply chain structure on supply chains with uncertain demand.

Demand uncertainty is one of the toughest challenges to supply chain management. A large body of literature on supply chain management has only involved one-period stochastic demand. Sana [20] studied the optimal order size for an inventory system under stochastic and price-dependent demand. Hsieh et al. [11] explored the pricing and ordering decisions for a multi-manufacturer and one-retailer supply chain system facing uncertain demand. Giri and Sharma [10] investigated the optimal manufacturing quantity for a closed-loop supply chain inventory system under stochastic demand, random return of used item, random yield rate and supply disruptions. Modak [17] considered the price and stocking decisions for the retail and online channels as well as the delivery lead time decision of online channel under price and delivery time sensitive additive stochastic demand. Jadidi et al. [13] addressed the joint pricing and ordering decision problem with transport capacity and cost for a supply chain with stochastic and price-dependent demand, where the dominant manufacturer offers all-unit quantity discounts to entice the buyer to order the items in full truckloads. Modak and Kelle [18] analyzed the optimal price, order quantity and lead time for a dual-channel supply chain under stochastic price and delivery-time dependent demand. Bhattacharyya and Sana [4] optimized the green technology, the capital invested for setup and the service level for a production inventory system of green products to achieve the maximum expected profit under stochastic and service level dependent demand. Modak and Kelle [19] studied the impacts of social responsibility, recycling, and carbon emissions on a closed-loop socially responsible supply chain, in which stochastic demand depends on sales price and amount of charitable donation announced for the society. Wang and Song [25] analyzed the selling prices of green and non-green products, sales effort and green level for a dual-channel supply chain under uncertain demand.

Recently, researchers began dealing with the problem of supply chain management under multi-period stochastic demand. Li et al. [14] analyzed the optimal normal order policy for the buyer and the optimal supplementary 
supply policy for the supplier, and properly design the supplementary supply order mechanism to enhance the supply chain performance in a multi-period setting. Zhang et al. [29] investigated a finite horizon coordinated pricing and inventory control problem under fixed ordering cost and capacity constraint, in which demand is price sensitive in an additive format. Lu and Song [15] characterized the optimal policies for single-period and multi-period inventory systems, respectively, where the production cost includes a fixed cost and a piecewise linear convex variable cost. Lu et al. [16] put forward a new approach called $K$-approximate convexity to study the multi-period joint pricing and inventory control problem with incomplete demand information and a nonconcave revenue function. Feinberg and Liang [9] explored the structure of the optimal inventory policies for a multi-period inventory control system with fixed ordering costs and all possible values of discount factor under both finite and infinite horizons. Song et al. [22] build a dynamic multi-period optimization model that incorporates reference price and customer loss aversion to analyze the optimal inventory level, replenishment quantity and the corresponding price for a finite horizon inventory control system. Benjaafar et al. [3] explored the optimal policies for a multi-period inventory control system with concave ordering costs. However, none of the above papers consider put option contract and two supply chain structures.

In supply chain management, high demand uncertainty is dealt with by a risk hedging contract. This paper mainly focuses on put option contract. Chen and Parlar [5] explored how a risk-averse newsvendor with a quadratic utility function decides the strike price and/or the strike quantity of put options to improve the riskreturn profile. Xue et al. [27] applied the conditional value-at-risk downside risk measure to study the optimal ordering and hedging policies for a risk-averse newsvendor with put option contract to hedge against the risk of low demand and maximize his expected utility. Wang and Chen [24] analyzed the joint ordering and pricing decision problem for a fresh produce newsvendor with price sensitive demand and the circulation loss of fresh produce under put option contract. Basu et al. [2] considered the impact of put option contract on a supply chain consisted of a risk-neutral supplier and a risk-averse retailer compared to the case with buyback contract. They showed that buyback contract can optimize the supply chain performance when the retailer's risk type is observable, but put option buyback contract can maximize the total supply chain profit when the retailer's risk type is unobservable. Wan and Chen [23] examined the value of put option contract on the decisions and performances of a supply chain under inflation. Hu et al. [12] introduced put option contract into a relief supply chain with a government and a supplier. They proved the superiority of put option contract over both wholesale price contract and buyback contract, and then derive a feasible range of the put option premium to attain the coordination of the relief supply chain. However, none of the above papers involve the multi-period setting and two supply chain structures.

Supply chain structure is an important issue in supply chain management with stochastic demand. Shi et al. [21] investigated the impact of supply chain structure on supply chains with stochastic and price-dependent demand. They suggested that the impact of supply chain structure on one firm's performance depends on the expected demand model, not the demand shock model. However, the impact of supply chain structure on the supply chain's efficiency depends on the models of expected demand and demand shock. Xue et al. [26] explored the impact of supply chain structure on channel performance and consumer surplus under random and price sensitive demand. They indicated that the order/production quantity is highest, the retail price is lowest and the expected surplus for an individual customer is largest when the manufacturer is the dominator. However, the channel profit and the expected surplus for the total consumers are highest when the retailer is the dominator. Chen et al. [6] analyzed the impact of supply chain structure on assembly supply chains consisted of one assembler and two suppliers under uncertain demand. They found that the assembler's profit is highest when the assembler is the most powerful, while the assembler with more power is not always better off when the assembler is not the most powerful. Similarly, the supplier's profit can decrease with more power he has. Yang et al. [28] examined the impacts of the firms' risk-averse attitudes and supply chain structure on the supply chain performance. They showed that when the supplier is more risk averse than the retailer, the manufacturer-dominated structure can lead to a higher optimal order quantity than the retailer-dominated structure. Meanwhile, the retailer-dominated structure cannot always outperform the manufacturer-dominated structure. However, none of the above papers consider the multi-period setting and put option contract. 
Although many works have been done in each field, few studies have incorporated the above aspects within a same research framework. This paper contributes the existing literature by introducing put option contract and two supply chain structures into a multi-period supply chain. One novelty of this paper is to analyze how the supplier and the manufacturer decide their multi-period production and ordering solutions with put option contract and two supply chain structures. In addition to the impact of put option contract, this paper investigates the impact of two supply chain structures from the perspectives of the members' decisions and total profits as well as the channel's total profit, which is another novelty of this paper. This paper fills the gap in the literature.

\section{Model DESCRIPTION AND ASSUMPtions}

This paper considers a two-stage supply chain consisted of a supplier and a manufacturer. The supplier produces a type of key components for the manufacturer who faces random demand. The manufacturer tailors the components into the final products to satisfy the customer needs. The selling horizon of final products is divided into multiple periods, indexed as $k=1, \ldots, n$. The supplier and the manufacturer are risk neutral and seek to maximize their own expected total profits.

This paper begins with a benchmark model without put option contract as the evaluation criterion, where wholesale price contract is used for protecting the manufacturer from demand risk. As is well known, wholesale price contract is characterized by one parameter, viz., the wholesale price. In each period $k$, the manufacturer orders a certain number of components from the supplier before observing the demand. The per unit price charged by the supplier for the order quantity of components is $w_{k}$. In order to hedge against demand uncertainty, put option contract is introduced by either the supplier or the manufacturer. It is all known that put option contract has a two-part fee structure, viz., the option price and the exercise price. In each period $k$, the manufacturer orders a certain number of put options from the supplier before observing the demand. The per unit price charged by the supplier for the order quantity of put options is $o_{k}$. If demand is lower than forecast, the manufacturer claims a refund from the supplier after observing the demand. The per unit price claimed from the supplier for the exercised quantity of put options is $e_{k}$.

The purpose of this paper is to study how put option contract and supply chain structure influence the members' decisions and total profits as well as the channel's total profit in the multi-period setting. Following the benchmark model without put option contract, this paper proposes two models with put option contract under two supply chain structures, respectively. In the manufacturer-led model with put option contract, the manufacturer behaviors as the leader of the channel and offers put option contract to the supplier. In the supplier-led model with put option contract, the supplier plays as the leader of the channel and offers put option contract to the manufacturer.

Additional notations used in this paper are introduced as follows.

\section{Parameters}

$D_{k} \quad$ Demand of final products in period $k$.

$F_{k}(\cdot) \quad$ The cumulative distribution function $(\mathrm{CDF})$ of $D_{k}$.

$f_{k}(\cdot) \quad$ The probability density function (PDF) of $D_{k}$.

$\mu_{k} \quad$ The expected value of $D_{k}$.

$\sigma_{k} \quad$ The standard variation of $D_{k}$.

$p_{k} \quad$ Unit retail price of final products in period $k$.

$b_{k} \quad$ Unit assembly cost of final products in period $k$.

$w_{k} \quad$ Unit wholesale price of components in period $k$.

$o_{k} \quad$ Unit option price in period $k$.

$e_{k} \quad$ Unit exercise price in period $k$.

$r_{k} \quad$ Unit urgent ordering cost of components in period $k$.

$h_{s k} \quad$ Unit holding cost of leftover components at the supplier's site in period $k$.

$h_{m k} \quad$ Unit holding cost of leftover components at the manufacturer's site in period $k$. 


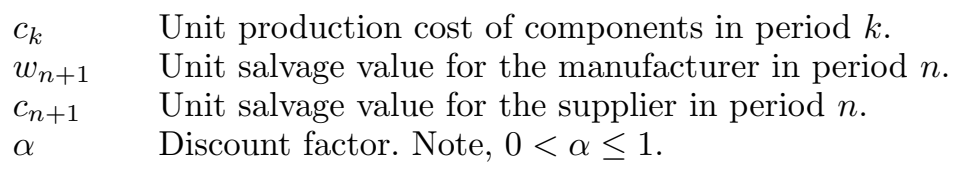

\section{State variables}

$L_{m k} \quad$ Initial inventory level of the manufacturer in period $k$.

$L_{s k} \quad$ Initial inventory level of the supplier in period $k$

$L_{c k} \quad$ Initial inventory level of the channel in period $k$.

\section{Decision variables}

$q_{w k} \quad$ The order quantity of components in period $k$.

$q_{m k} \quad$ The order quantity of put options in period $k$.

$q_{s k} \quad$ The production quantity in period $k$.

$\bar{y}_{k} \quad$ The maximum available quantity of components to satisfy demand in period $k$.

$\underline{y}_{k} \quad$ The minimum available quantity of components to satisfy demand in period $k$.

\section{Other notations}

$V_{m k}(\cdot)$ The profit-to-go function of the manufacturer.

$V_{s k}(\cdot) \quad$ The profit-to-go function of the supplier.

$V_{c k}(\cdot) \quad$ The profit-to-go function of the channel.

$\Pi_{m}(\cdot) \quad$ The total profit of the manufacturer.

$\Pi_{s}(\cdot) \quad$ The total profit of the supplier.

$\Pi_{c}(\cdot) \quad$ The total profit of the channel, where $\Pi_{c}(\cdot)=\Pi_{m}(\cdot)+\Pi_{s}(\cdot)$

$[\chi]^{+} \quad$ Maximum value of 0 and $\chi$.

$\chi^{*} \quad$ Optimal value of $\chi$.

$\chi^{+} \quad$ Myopic value of $\chi$.

Other notations are defined when they are needed. Throughout the paper, the supplier is viewed as a "male" and the manufacturer is viewed as a "female". The superscripts " $\mathcal{D}$ ", " $\mathcal{P}$ ", " $\mathcal{R}$ " and " $\mathcal{J}$ " stand for "the benchmark model", "the manufacturer-led model", "the supplier-led model" and "the integrated model", respectively.

Without loss of generality, the assumption of the model is given as follows.

(1) The relationship between the component and the product is 1:1 [8].

(2) Demand information is symmetry between the supplier and the manufacturer.

(3) Demands for sequential periods are independent and identically distributed.

(4) In each period $k$, the manufacturer has an chance to buy from an alternative source by paying a higher ordering cost $r_{k}$ if demand is higher than forecast.

(5) $r_{k}>w_{k}>c_{k}$. This condition ensures that the wholesale price is higher than the production cost, but is lower than the urgent ordering cost.

(6) $w_{k}>e_{k}-o_{k}$. This condition prevents the manufacturer from arbitraging by buying the put options.

(7) $c_{k}+h_{s k}-\alpha c_{k+1}>w_{k}+o_{k}-e_{k}$. This condition prevents the supplier from producing an inordinate quantity of components.

(8) $e_{k}+h_{m k}-\alpha w_{k+1}>o_{k}$. This condition ensures the buying intention of the manufacturer on the put options.

(9) $h_{m k}>h_{s k}$. This condition ensures that the holding cost of leftover components at the supplier's site is lower than the holding cost of leftover components at the manufacturer's site.

\section{Benchmark Model Without put option CONTRACT}

To illustrate the impact of put option contract, we first analyze the benchmark model without put option contract as the evaluation criterion. Following Eriksson [8], the sequence of events is as follows. Before the beginning of period $k$, the supplier offers the manufacturer the wholesale price contract and decides the production quantity $q_{s k}^{\mathcal{D}}$. Based on the contract terms and the supplier's production strategy, the manufacturer decides the 
order quantity of components $q_{w k}^{\mathcal{D}}$. At the beginning of period $k$, the supplier delivers the components to the manufacturer immediately. During period $k$, the components are tailored by the manufacturer into the final products so as to meet the market demand. If demand is higher than forecast, insufficient components are replenished by an urgent order from an alternative source. At the end of period $k$, redundant components are carried over to next period until the last period of the selling horizon.

\subsection{The manufacturer's optimal ordering policy}

For period $k=1, \ldots, n$, when the manufacturer's component order is placed, the maximum available quantity of components to satisfy demand is $\bar{y}_{k}^{\mathcal{D}}=L_{m k}^{\mathcal{D}}+q_{w k}^{\mathcal{D}}$. Thus, the inventory levels for the manufacturer in two successive periods are related as $L_{m k+1}^{\mathcal{D}}=E\left[\left(\bar{y}_{k}^{\mathcal{D}}-D_{k}\right)^{+}\right]$. The manufacturer's expected total profit over all periods is

$$
\Pi_{m}^{\mathcal{D}}\left(\bar{y}_{1}^{\mathcal{D}}, \ldots, \bar{y}_{n}^{\mathcal{D}}\right)=\sum_{k=1}^{n}\left\{\left(p_{k}-b_{k}\right) E\left(D_{k}\right)-w_{k}\left(\bar{y}_{k}^{\mathcal{D}}-L_{m k}^{\mathcal{D}}\right)-r_{k} E\left[\left(D_{k}-\bar{y}_{k}^{\mathcal{D}}\right)^{+}\right]-h_{m k} E\left[\left(\bar{y}_{k}^{\mathcal{D}}-D_{k}\right)\right]\right\} .
$$

Let $V_{m k}^{\mathcal{D}}\left(L_{m k}^{\mathcal{D}}\right)$ be the profit-to-go function for the manufacturer with initial state $L_{m k}^{\mathcal{D}}$. Then, the recursion can be formulated as follows.

$$
V_{m k}^{\mathcal{D}}\left(L_{m k}^{\mathcal{D}}\right)=w_{k} L_{m k}^{\mathcal{D}}+\max _{\bar{y}_{k}^{\mathcal{D}} \geq L_{m k}^{\mathcal{D}}}\left\{\pi_{m k}^{\mathcal{D}}\left(\bar{y}_{k}^{\mathcal{D}}\right)+\alpha E V_{m k+1}^{\mathcal{D}}\left[\left(\bar{y}_{k}^{\mathcal{D}}-D_{k}\right)^{+}\right]\right\}
$$

where $V_{m n+1}^{\mathcal{D}}\left(L_{m n+1}^{\mathcal{D}}\right)=w_{n+1} L_{m n+1}^{\mathcal{D}}$ and

$$
\pi_{m k}^{\mathcal{D}}\left(\bar{y}_{k}^{\mathcal{D}}\right)=\left(p_{k}-b_{k}\right) E\left(D_{k}\right)-w_{k} \bar{y}_{k}^{\mathcal{D}}-r_{k} E\left[\left(D_{k}-\bar{y}_{k}^{\mathcal{D}}\right)^{+}\right]-h_{m k} E\left[\left(\bar{y}_{k}^{\mathcal{D}}-D_{k}\right)^{+}\right] .
$$

In equation (4.3), the first term is the incomes and the assembly costs from the sales of final products, the second term is the wholesale ordering costs of components, the third term is the urgent ordering costs of components and the last term is the holding costs of components.

Let $V_{m k}^{\mathcal{D}+}\left(L_{m k}^{\mathcal{D}}\right)=V_{m k}^{\mathcal{D}}\left(L_{m k}^{\mathcal{D}}\right)-w_{k} L_{m k}^{\mathcal{D}}$. The recursion can be reformulated as follows.

$$
V_{m k}^{\mathcal{D}+}\left(L_{m k}^{\mathcal{D}}\right)=\max _{\bar{y}_{k}^{\mathcal{D}} \geq L_{m k}^{\mathcal{D}}}^{\mathcal{D}}\left\{\pi_{m k}^{\mathcal{D}+}\left(\bar{y}_{k}^{\mathcal{D}}\right)+\alpha E V_{m k+1}^{\mathcal{D}+}\left[\left(\bar{y}_{k}^{\mathcal{D}}-D_{k}\right)^{+}\right]\right\}
$$

where $V_{m n+1}^{\mathcal{D}+}\left(L_{m n+1}^{\mathcal{D}}\right)=0$ and

$$
\begin{aligned}
\pi_{m k}^{\mathcal{D}+}\left(\bar{y}_{k}^{\mathcal{D}}\right)= & \left(p_{k}-b_{k}\right) E\left(D_{k}\right)-w_{k} \bar{y}_{k}^{\mathcal{D}}-r_{k} E\left[\left(D_{k}-\bar{y}_{k}^{\mathcal{D}}\right)^{+}\right]-h_{m k} E\left[\left(\bar{y}_{k}^{\mathcal{D}}-D_{k}\right)^{+}\right] \\
& +\alpha w_{k+1} E\left[\left(\bar{y}_{k}^{\mathcal{D}}-D_{k}\right)^{+}\right] .
\end{aligned}
$$

In equation (4.5), the first four terms are the manufacturer's profit margin in current period and the last term is the discounted value of leftover components at the manufacturer's site in next period. Based on the above analysis, we obtain the following lemma.

Lemma 4.1. For period $k=1, \ldots, n, V_{m k}^{\mathcal{D}+}\left(L_{m k}^{\mathcal{D}}\right)$ is a concave function of $L_{m k}^{\mathcal{D}}$.

This lemma indicates that in the benchmark model there always exists a unique optimal ordering decision for the manufacturer in each period. As to the specific solution, we have the following proposition.

Proposition 4.2. In the benchmark model, for period $k=1, \ldots, n$, the optimal component order quantity of the manufacturer is

$$
q_{w k}^{\mathcal{D} *}= \begin{cases}S_{k}^{\mathcal{D} *}-L_{m k}^{\mathcal{D}} & \text { if } L_{m k}^{\mathcal{D}} \leq S_{k}^{\mathcal{D} *} \\ 0 & \text { if } L_{m k}^{\mathcal{D}}>S_{k}^{\mathcal{D} *}\end{cases}
$$


where $S_{k}^{\mathcal{D} *}=\arg \max _{\bar{y}_{k}^{\mathcal{D}}}\left\{\pi_{m k}^{\mathcal{D}+}\left(\bar{y}_{k}^{\mathcal{D}}\right)+\alpha E V_{m k+1}^{\mathcal{D}+}\left[\left(\bar{y}_{k}^{\mathcal{D}}-D_{k}\right)^{+}\right]\right\}$.

This proposition suggests that in the benchmark model the optimal ordering policy of the manufacturer in period $k$ depends on the initial state $L_{m k}^{\mathcal{D}}$ and the optimal policy $S_{k}^{\mathcal{D} *}$ in period $k$. The manufacturer should guarantee the maximum available quantity of components that satisfies demand to be not less than $S_{k}^{\mathcal{D} *}$ in period $k$. As to the impact of the wholesale price on the optimal policy $S_{k}^{\mathcal{D} *}$, we get the following corollary.

Corollary 4.3. For period $k=1, \ldots, n, S_{k}^{\mathcal{D} *}$ is decreasing in $w_{k}$.

This corollary illustrates that in the benchmark model the optimal policy $S_{k}^{\mathcal{D} *}$ in period $k$ is negatively affected by the wholesale price in period $k$. That is, an increase in the wholesale price in period $k$ may induce a decreased component order quantity for the manufacturer in period $k$.

As stated by Li et al. [14], it is not easy to calculate the exact value of optimal policy. In the following, a myopic approach is provided to make the policy more straightforward to be implemented. It is worth highlighting that the myopic policy is optimal in the event that all the parameters, i.e., the costs, the prices and the demands, are stationary over all periods. We denote $S_{k}^{\mathcal{D}+}$ as a myopic policy for the manufacturer's component order when the effect of the manufacturer's current ordering decision on her future profit can be ignored. Then, we have following proposition.

Proposition 4.4. For period $k=1, \ldots, n$, we have $S_{k}^{\mathcal{D}+}=F_{k}^{-1}\left(\frac{r_{k}-w_{k}}{r_{k}+h_{m k}-\alpha w_{k+1}}\right)$

This proposition shows that in the benchmark model the myopic policy $S_{k}^{\mathcal{D}+}$ in period $k$ is negatively affected by the wholesale price in period $k$, which is in line with the result of Corollary 4.3. However, we find that in the benchmark model the myopic policy $S_{k}^{\mathcal{D}+}$ in period $k$ is positively affected by the wholesale price in period $k+1$. That is, an increase in the wholesale price in period $k+1$ may induce an increased component order quantity for the manufacturer in period $k$.

\subsection{The supplier's optimal production policy}

For period $k=1, \ldots, n$, the supplier produces the components up to satisfying the required component order quantity of the manufacturer, that is, $q_{s k}^{\mathcal{D}}=q_{w k}^{\mathcal{D} *}$. The supplier's expected total profit over all periods is

$$
\Pi_{s}^{\mathcal{D}}\left(\bar{y}_{1}^{\mathcal{D} *}, \ldots, \bar{y}_{n}^{\mathcal{D} *}\right)=\sum_{k=1}^{n}\left(w_{k}-c_{k}\right)\left(\bar{y}_{k}^{\mathcal{D} *}-L_{m k}^{\mathcal{D}}\right) .
$$

It is easy to see that the supplier adopts the make-to-order production strategy so that the demand risk is fully undertaken by the manufacturer.

\section{Two MODELS With PUT OPTION CONTRACT}

Now we study the models with put option contract under two supply chain structures, i.e., the manufacturerled model with put option contract and the supplier-led model with put option contract.

\subsection{Manufacturer-led model with put option contract}

In the manufacturer-led model, the manufacturer is the leader and the supplier is the follower. Following Arani et al. [1], the sequence of events is as follows. Before the beginning of period $k$, the manufacturer offers the supplier the wholesale price and put option portfolio contract and decides the order quantity of put options $q_{m k}^{\mathcal{P}}$. Based on the contract terms and the manufacturer's ordering strategy, the supplier decides the production quantity $q_{s k}^{\mathcal{P}}$. The manufacturer commits to pay the supplier per unit wholesale price for the reserved capacity. At the beginning of period $k$, the supplier delivers the components to the manufacturer's site immediately. During period $k$, the components are assembled by the manufacturer into the final products so as to meet the market demand. If demand is higher than forecast, insufficient components are replenished by an urgent order 
from an alternative source. If demand is lower than forecast, the put options are exercised by the manufacturer to send back part of redundant components to the supplier. At the end of period $k$, redundant components are carried over to next period until the last period of the selling horizon.

\subsection{The supplier's optimal production policy}

For period $k=1, \ldots, n$, when the supplier's production is completed, the maximum available quantity of components to satisfy demand is $\bar{y}_{k}^{\mathcal{P}}=L_{m k}^{\mathcal{P}}+L_{s k}^{\mathcal{P}}+q_{s k}^{\mathcal{P}}$; when all the put options are exercised, the minimum available quantity of components to satisfy demand is $\underline{y}_{k}^{\mathcal{P}}=\bar{y}_{k}^{\mathcal{P}}-q_{m k}^{\mathcal{P}}$. So, the inventory levels for the supplier in two successive periods are related as $L_{s k+1}^{\mathcal{P}}=E \min \left[\left(\bar{y}_{k}^{\mathcal{P}}-\underline{y}_{k}^{\mathcal{P}}\right),\left(\bar{y}_{k}^{\mathcal{P}}-D_{k}\right)^{+}\right]$. The supplier's expected total profit over all periods is

$$
\begin{aligned}
\Pi_{s}^{\mathcal{P}}\left(\bar{y}_{1}^{\mathcal{P}}, \ldots, \bar{y}_{n}^{\mathcal{P}}\right)= & \sum_{k=1}^{n}\left\{w_{k}\left(\bar{y}_{k}^{\mathcal{P}}-L_{m k}^{\mathcal{P}}\right)+o_{k}\left(\bar{y}_{k}^{\mathcal{P}}-\underline{y}_{k}^{\mathcal{P}}\right)-\left(e_{k}+h_{s k}\right) E \min \left[\left(\bar{y}_{k}^{\mathcal{P}}\right.\right.\right. \\
& \left.\left.\left.-\underline{y}_{k}^{\mathcal{P}}\right),\left(\bar{y}_{k}^{\mathcal{P}}-D_{k}\right)^{+}\right]-c_{k}\left(\bar{y}_{k}^{\mathcal{P}}-L_{m k}^{\mathcal{P}}-L_{s k}^{\mathcal{P}}\right)\right\}
\end{aligned}
$$

Let $V_{s k}^{\mathcal{P}}\left(L_{s k}^{\mathcal{P}}\right)$ be the profit-to-go function for the supplier with initial state $L_{s k}^{\mathcal{P}}$. Then, the recursion can be formulated as follows.

$$
V_{s k}^{\mathcal{P}}\left(L_{s k}^{\mathcal{P}}\right)=c_{k} L_{s k}^{\mathcal{P}}+\max _{\bar{y}_{k}^{\mathcal{P}} \geq \underline{y}_{k}^{\mathcal{P}}}\left\{\pi_{s k}^{\mathcal{P}}\left(\bar{y}_{k}^{\mathcal{P}}\right)+\alpha E V_{s k+1}^{\mathcal{P}}\left(\min \left[\left(\bar{y}_{k}^{\mathcal{P}}-\underline{y}_{k}^{\mathcal{P}}\right),\left(\bar{y}_{k}^{\mathcal{P}}-D_{k}\right)^{+}\right]\right)\right\}
$$

where $V_{s n+1}^{\mathcal{P}}\left(L_{s n+1}^{\mathcal{P}}\right)=c_{n+1} L_{s n+1}^{\mathcal{P}}$ and

$$
\begin{aligned}
\pi_{s k}^{\mathcal{P}}\left(\bar{y}_{k}^{\mathcal{P}}\right)= & w_{k}\left(\bar{y}_{k}^{\mathcal{P}}-L_{m k}^{\mathcal{P}}\right)+o_{k}\left(\bar{y}_{k}^{\mathcal{P}}-\underline{y}_{k}^{\mathcal{P}}\right)-\left(e_{k}+h_{s k}\right) E \min \left[\left(\bar{y}_{k}^{\mathcal{P}}-\underline{y}_{k}^{\mathcal{P}}\right),\left(\bar{y}_{k}^{\mathcal{P}}-D_{k}\right)^{+}\right] \\
& -c_{k}\left(\bar{y}_{k}^{\mathcal{P}}-L_{m k}^{\mathcal{P}}\right) .
\end{aligned}
$$

In equation (5.3), the first term is the incomes from the wholesales of components, the second term is the incomes from the sales of put options, the third term is the costs of exercising the put options and holding the components and the last term is the production costs.

Let $V_{s k}^{\mathcal{P}+}\left(L_{s k}^{\mathcal{P}}\right)=V_{s k}^{\mathcal{P}}\left(L_{s k}^{\mathcal{P}}\right)-c_{k} L_{s k}^{\mathcal{P}}$. The recursion can be reformulated as follows.

$$
V_{s k}^{\mathcal{P}+}\left(L_{s k}^{\mathcal{P}}\right)=\max _{\bar{y}_{k}^{\mathcal{P}} \geq \underline{y}_{k}^{\mathcal{P}}}\left\{\pi_{s k}^{\mathcal{P}+}\left(\bar{y}_{k}^{\mathcal{P}}\right)+\alpha E V_{s k+1}^{\mathcal{P}+}\left(\min \left[\left(\bar{y}_{k}^{\mathcal{P}}-\underline{y}_{k}^{\mathcal{P}}\right),\left(\bar{y}_{k}^{\mathcal{P}}-D_{k}\right)^{+}\right]\right)\right\}
$$

where $V_{s n+1}^{\mathcal{P}+}\left(L_{s n+1}^{\mathcal{P}}\right)=0$ and

$$
\begin{aligned}
\pi_{s k}^{\mathcal{P}+}\left(\bar{y}_{k}^{\mathcal{P}}\right)= & \left(w_{k}+o_{k}-c_{k}\right)\left(\bar{y}_{k}^{\mathcal{P}}-\underline{y}_{k}^{\mathcal{P}}\right)-\left(e_{k}+h_{s k}\right) E \min \left[\left(\bar{y}_{k}^{\mathcal{P}}-\underline{y}_{k}^{\mathcal{P}}\right),\left(\bar{y}_{k}^{\mathcal{P}}-D_{k}\right)^{+}\right] \\
& +\left(w_{k}-c_{k}\right)\left(\underline{y}_{k}^{\mathcal{P}}-L_{m k}^{\mathcal{P}}\right)+\alpha c_{k+1} E \min \left[\left(\bar{y}_{k}^{\mathcal{P}}-\underline{y}_{k}^{\mathcal{P}}\right),\left(\bar{y}_{k}^{\mathcal{P}}-D_{k}\right)^{+}\right]
\end{aligned}
$$

In equation (5.5), the first three terms are the supplier's profit margin in current period and the last term is the discounted value of leftover components at the supplier's site in next period. Based on the above analysis, we obtain the following lemma.

Lemma 5.1. For period $k=1, \ldots, n, V_{s k}^{\mathcal{P}+}\left(L_{s k}^{\mathcal{P}}\right)$ is a concave function of $L_{s k}^{\mathcal{P}}$.

This lemma indicates that in the manufacturer-led model there always exists a unique optimal production decision for the supplier in each period. As to the specific solution, we have the following proposition. 
Proposition 5.2. In the manufacturer-led model, for period $k=1, \ldots, n$, the optimal production quantity of the supplier is

$$
q_{s k}^{\mathcal{P} *}= \begin{cases}S_{k}^{\mathcal{P} *}-L_{m k}^{\mathcal{P}}-L_{s k}^{\mathcal{P}} & \text { if } \underline{y}_{k}^{\mathcal{P}} \leq S_{k}^{\mathcal{P} *} \\ \left(\underline{y}_{k}^{\mathcal{P}}-L_{m k}^{\mathcal{P}}-L_{s k}^{\mathcal{P}}\right)^{+} & \text {if } \underline{y}_{k}^{\mathcal{P}}>S_{k}^{\mathcal{P} *}\end{cases}
$$

where $S_{k}^{\mathcal{P} *}=\arg \max _{\bar{y}_{k}^{\mathcal{P}}}\left\{\pi_{s k}^{\mathcal{P}+}\left(\bar{y}_{k}^{\mathcal{P}}\right)+\alpha E V_{s k+1}^{\mathcal{P}+}\left(\min \left[\left(\bar{y}_{k}^{\mathcal{P}}-\underline{y}_{k}^{\mathcal{P}}\right),\left(\bar{y}_{k}^{\mathcal{P}}-D_{k}\right)^{+}\right]\right)\right\}$.

This proposition shows that in the manufacturer-led model the optimal production policy of the supplier in period $k$ depends on the manufacturer's decision $\underline{y}_{k}^{\mathcal{P}}$ and the optimal policy $S_{k}^{\mathcal{P} *}$ in period $k$. The supplier should guarantee the maximum available quantity of components that satisfies demand to be not less than $S_{k}^{\mathcal{P} *}$ in period $k$. As to the impact of the contract parameters on the optimal policy $S_{k}^{\mathcal{P} *}$, we get the following corollary.

Corollary 5.3. For period $k=1, \ldots, n, S_{k}^{\mathcal{P} *}$ is increasing in $w_{k}$ and $o_{k}$, but is decreasing in $e_{k}$.

This corollary illustrates in the manufacturer-led model the optimal policy $S_{k}^{\mathcal{P} *}$ in period $k$ is positively affected by the wholesale price and the option price in period $k$, while is negatively affected by the exercise price in period $k$. That is, an increase in the wholesale price or the option price in period $k$ may induce an increased production quantity for the supplier in period $k$. However, an increase in the exercise price in period $k$ may induce a decreased production quantity for the supplier in period $k$.

We denote $S_{k}^{\mathcal{P}+}$ as a myopic policy for the supplier's production when the effect of the supplier's current production decision on his future profit can be ignored. Then, we have the following proposition.

Proposition 5.4. For period $k=1, \ldots, n$, we have $S_{k}^{\mathcal{P}+}=F_{k}^{-1}\left(\frac{w_{k}+o_{k}-c_{k}}{e_{k}+h_{s k}-\alpha c_{k+1}}\right)$

This proposition suggests that in the manufacturer-led model the myopic policy $S_{k}^{\mathcal{P}+}$ in period $k$ is negatively affected by the production cost in period $k$, but is positively affected by the production cost in period $k+1$. That is, an increase in the production cost in period $k$ may induce an decreased production quantity for the supplier in period $k$. However, an increase in the production cost in period $k+1$ may induce an increased production quantity for the supplier in period $k$.

\subsection{The manufacturer's optimal ordering policy}

For period $k=1, \ldots, n$, the inventory levels for the manufacturer in two successive periods are related as $L_{m k+1}^{\mathcal{P}}=E\left[\left(\underline{y}_{k}^{\mathcal{P}}-D_{k}\right)^{+}\right]$. The manufacturer's expected total profit over all periods is

$$
\begin{aligned}
\Pi_{m}^{\mathcal{P}}\left(\underline{y}_{1}^{\mathcal{P}}, \ldots, \underline{y}_{n}^{\mathcal{P}}\right)= & \sum_{k=1}^{n}\left\{\left(p_{k}-b_{k}\right) E\left(D_{k}\right)-w_{k}\left(\bar{y}_{k}^{\mathcal{P} *}-L_{m k}^{\mathcal{P}}\right)-o_{k}\left(\bar{y}_{k}^{\mathcal{P} *}-\underline{y}_{k}^{\mathcal{P}}\right)\right. \\
& +e_{k} E \min \left[\left(\bar{y}_{k}^{\mathcal{P} *}-\underline{y}_{k}^{\mathcal{P}}\right),\left(\bar{y}_{k}^{\mathcal{P} *}-D_{k}\right)^{+}\right]-r_{k} E\left[\left(D_{k}-\bar{y}_{k}^{\mathcal{P} *}\right)^{+}\right] \\
& \left.-h_{m k} E\left[\left(\underline{y}_{k}^{\mathcal{P}}-D_{k}\right)^{+}\right]\right\} .
\end{aligned}
$$

Let $V_{m k}^{\mathcal{P}}\left(L_{m k}^{\mathcal{P}}\right)$ be the profit-to-go function for the manufacturer with initial state $L_{m k}^{\mathcal{P}}$. Then, the recursion can be formulated as follows.

$$
V_{m k}^{\mathcal{P}}\left(L_{m k}^{\mathcal{P}}\right)=w_{k} L_{m k}^{\mathcal{P}}+\max _{\underline{y}_{k}^{\mathcal{P}} \geq L_{m k}^{\mathcal{P}}}\left\{\pi_{m k}^{\mathcal{P}}\left(\underline{y}_{k}^{\mathcal{P}}\right)+\alpha E V_{m k+1}^{\mathcal{P}}\left[\left(\underline{y}_{k}^{\mathcal{P}}-D_{k}\right)^{+}\right]\right\}
$$

where $V_{m n+1}^{\mathcal{P}}\left(L_{m n+1}^{\mathcal{P}}\right)=w_{n+1} L_{m n+1}^{\mathcal{P}}$ and

$$
\begin{aligned}
\pi_{m k}^{\mathcal{P}}\left(\underline{y}_{k}^{\mathcal{P}}\right)= & \left(p_{k}-b_{k}\right) E\left(D_{k}\right)-w_{k} \bar{y}_{k}^{\mathcal{P} *}-o_{k}\left(\bar{y}_{k}^{\mathcal{P} *}-\underline{y}_{k}^{\mathcal{P}}\right)+e_{k} E \min \left[\left(\bar{y}_{k}^{\mathcal{P} *}-\underline{y}_{k}^{\mathcal{P}}\right),\right. \\
& \left.\left(\bar{y}_{k}^{\mathcal{P} *}-D_{k}\right)^{+}\right]-r_{k} E\left[\left(D_{k}-\bar{y}_{k}^{\mathcal{P} *}\right)^{+}\right]-h_{m k} E\left[\left(\underline{y}_{k}^{\mathcal{P}}-D_{k}\right)^{+}\right] .
\end{aligned}
$$


In equation (5.8), the first term is the incomes and the assembly costs from the sale of final products, the second term is the wholesale ordering costs of components, the third terms is the purchasing costs of put options, the fourth term is the incomes from exercising the put options, the fifth term is the urgent ordering costs of components and the last term is the holding costs of components.

Let $V_{m k}^{\mathcal{P}+}\left(L_{m k}^{\mathcal{P}}\right)=V_{m k}^{\mathcal{P}}\left(L_{m k}^{\mathcal{P}}\right)-w_{k} L_{m k}^{\mathcal{P}}$. The recursion can be reformulated as follows.

$$
V_{m k}^{\mathcal{P}+}\left(L_{m k}^{\mathcal{P}}\right)=\max _{\underline{y}_{k}^{\mathcal{P}} \geq L_{m k}^{\mathcal{P}}}\left\{\pi_{m k}^{\mathcal{P}+}\left(\underline{y}_{k}^{\mathcal{P}}\right)+\alpha E V_{m k+1}^{\mathcal{P}+}\left[\left(\underline{y}_{k}^{\mathcal{P}}-D_{k}\right)^{+}\right]\right\}
$$

where $V_{m n+1}^{\mathcal{P}+}\left(L_{m n+1}^{\mathcal{P}}\right)=0$ and

$$
\begin{aligned}
\pi_{m k}^{\mathcal{P}+}\left(\underline{y}_{k}^{\mathcal{P}}\right)= & \left(p_{k}-b_{k}\right) E\left(D_{k}\right)-w_{k} \bar{y}_{k}^{\mathcal{P} *}-o_{k}\left(\bar{y}_{k}^{\mathcal{P} *}-\underline{y}_{k}^{\mathcal{P}}\right)+e_{k} E \min \left[\left(\bar{y}_{k}^{\mathcal{P} *}-\underline{y}_{k}^{\mathcal{P}}\right),\left(\bar{y}_{k}^{\mathcal{P} *}-D_{k}\right)^{+}\right] \\
& -r_{k} E\left[\left(D_{k}-\bar{y}_{k}^{\mathcal{P} *}\right)^{+}\right]-h_{m k} E\left[\left(\underline{y}_{k}^{\mathcal{P}}-D_{k}\right)^{+}\right]+\alpha w_{k+1} E\left[\left(\underline{y}_{k}^{\mathcal{P}}-D_{k}\right)^{+}\right] .
\end{aligned}
$$

In equation (5.10), the first six terms are the manufacturer's profit margin in current period and the last term is the discounted value of leftover components at the manufacturer's site in next period. Based on the above analysis, we obtain the following lemma.

Lemma 5.5. For period $k=1, \ldots, n, V_{m k}^{\mathcal{P}+}\left(L_{m k}^{\mathcal{P}}\right)$ is a concave function of $L_{m k}^{\mathcal{P}}$.

This lemma indicates that in the manufacturer-led model there always exists a unique optimal ordering decision for the manufacturer in each period. As to the specific solution, we have the following proposition.

Proposition 5.6. In the manufacturer-led model, for period $k=1, \ldots, n$, the optimal put options order quantity of the manufacturer is

$$
q_{m k}^{\mathcal{P} *}= \begin{cases}S_{k}^{\mathcal{P} *}-s_{k}^{\mathcal{P} *} & \text { if } L_{m k}^{\mathcal{P}} \leq s_{k}^{\mathcal{P} *} \\ S_{k}^{\mathcal{P} *}-L_{m k}^{\mathcal{P}} & \text { if } s_{k}^{\mathcal{P} *}<L_{m k}^{\mathcal{P}} \leq S_{k}^{\mathcal{P} *} \\ 0 & \text { if } L_{m k}^{\mathcal{P}}>S_{k}^{\mathcal{P} *}\end{cases}
$$

where $s_{k}^{\mathcal{P} *}=\arg \max _{\underline{y}_{k}^{\mathcal{P}}}\left\{\pi_{m k}^{\mathcal{P}+}\left(\underline{y}_{k}^{\mathcal{P}}\right)+\alpha E V_{m k+1}^{\mathcal{P}+}\left[\left(\underline{y}_{k}^{\mathcal{P}}-D_{k}\right)^{+}\right]\right\}$.

This proposition suggests that in the manufacturer-led model the optimal ordering policy of the manufacturer in period $k$ depends on the initial state $L_{m k}^{\mathcal{P}}$, the optimal policy $s_{k}^{\mathcal{P} *}$ and the optimal policy $S_{k}^{\mathcal{P} *}$ in period $k$. The manufacturer should guarantee the minimum available quantity of components that satisfies demand to be not less than $s_{k}^{\mathcal{P} *}$ in period $k$. As to the impact of the contract parameters on the optimal policy $s_{k}^{\mathcal{P} *}$, we get the following corollary.

Corollary 5.7. For period $k=1, \ldots, n, s_{k}^{\mathcal{P} *}$ is constant in $w_{k}$, is increasing in $o_{k}$, but is decreasing in $e_{k}$.

This corollary illustrate in the manufacturer-led model the optimal policy $s_{k}^{\mathcal{P} *}$ in period $k$ has nothing to do with the wholesale price in period $k$, while is positively affected by the option price in period $k$ and is negatively affected by the exercise price in period $k$. That is, an increase in the wholesale price in period $k$ may induce an increased put options order quantity for the manufacturer in period $k$. However, an increase in the option price or the exercise price in period $k$ may induce an inscrutable put options order quantity for the manufacturer in period $k$.

We denote $s_{k}^{\mathcal{P}+}$ as a myopic policy for the manufacturer's put options order when the effect of the manufacturer's current ordering decision on her future profit can be ignored. Then, we have the following proposition.

Proposition 5.8. For period $k=1, \ldots, n$, we have $s_{k}^{\mathcal{P}+}=F_{k}^{-1}\left(\frac{o_{k}}{e_{k}+h_{m k}-\alpha w_{k+1}}\right)$.

This proposition shows that in the manufacturer-led model the myopic policy $s_{k}^{\mathcal{P}+}$ in period $k$ is not affected by the wholesale price in period $k$, which is in line with the result of Corollary 5.7. However, we find that in the 
manufacturer-led model the myopic policy $s_{k}^{\mathcal{P}+}$ in period $k$ is positively affected by the wholesale price in period $k+1$. That is, an increase in the wholesale price in period $k+1$ may induce a decreased put options order quantity for the manufacturer in period $k$.

Note that $s_{k}^{\mathcal{P}+}<S_{k}^{\mathcal{P}+}$. This means that in the manufacturer-led model the key decision for two members is to ensure that the minimum available quantity of components always fall below the available maximum quantity of components in each period. This inequality equals to $o_{k}<\frac{\left(w_{k}-c_{k}\right)\left(e_{k}+h_{m k}-\alpha w_{k+1}\right)}{\left(h_{s k}-\alpha c_{k+1}\right)-\left(h_{m k}-\alpha w_{k+1}\right)}$, which indicates that the option price should be not too high.

\subsection{Supplier-led model with put option contract}

In the supplier-led model, the supplier is the leader and the manufacturer is the follower. Following Wan and Chen [23], the sequence of events is as follows. Before the beginning of period $k$, the supplier offers the manufacturer the wholesale price and put option portfolio contract and decides the production quantity $q_{s k}^{\mathcal{R}}$. Based on the contract terms and the supplier's production strategy, the manufacturer simultaneously decides the order quantity of components $q_{w k}^{\mathcal{R}}$ and the order quantity of put options $q_{m k}^{\mathcal{R}}$. At the beginning of period $k$, the supplier delivers the components to the manufacturer's site immediately. During period $k$, the components are assembled by the manufacturer into the final products so as to meet the market demand. If demand is higher than forecast, insufficient components are replenished by an urgent order from an alternative source. If demand is lower than forecast, the put options are exercised by the manufacturer to send back part of redundant components to the supplier. At the end of period $k$, redundant components are carried over to next period until the last period of the selling horizon.

\subsection{The manufacturer's optimal production policy}

For period $k=1, \ldots, n$, when the manufacturer's component order and put options order are placed, the maximum available quantity of components to satisfy demand is $\bar{y}_{k}^{\mathcal{R}}=L_{m k}^{\mathcal{R}}+q_{w k}^{\mathcal{R}}$; when all the put options are exercised, the minimum available quantity of components to satisfy demand is $\underline{y}_{k}^{\mathcal{R}}=\bar{y}_{k}^{\mathcal{R}}-q_{m k}^{\mathcal{R}}$. Therefore, the inventory levels for the manufacturer in two successive periods are related as $L_{m k}^{\mathcal{R}}=E\left[\left(\underline{y}_{k}^{\mathcal{R}}-D_{k}\right)^{+}\right]$. The manufacturer's expected total profit over all periods is

$$
\begin{aligned}
\Pi_{m}^{\mathcal{R}}\left(\bar{y}_{1}^{\mathcal{R}}, \ldots, \bar{y}_{n}^{\mathcal{R}} ; \underline{y}_{1}^{\mathcal{R}}, \ldots, \bar{y}_{n}^{\mathcal{R}}\right)= & \sum_{k=1}^{n}\left\{\left(p_{k}-b_{k}\right) E\left(D_{k}\right)-w_{k}\left(\bar{y}_{k}^{\mathcal{R}}-L^{\mathcal{R}_{m k}}\right)-o_{k}\left(\bar{y}_{k}^{\mathcal{R}}-\underline{y}_{k}^{\mathcal{R}}\right)\right. \\
& +e_{k} E\left[\min \left(\bar{y}_{k}^{\mathcal{R}}-\underline{y}_{k}^{\mathcal{R}}\right),\left(\bar{y}_{k}^{\mathcal{R}}-D_{k}\right)^{+}\right]-r_{k} E\left[\left(D_{k}-\bar{y}_{k}^{\mathcal{R}}\right)^{+}\right] \\
& \left.-h_{m k} E\left[\left(\underline{y}_{k}^{\mathcal{R}}-D_{k}\right)^{+}\right]\right\} .
\end{aligned}
$$

Let $V_{m k}^{\mathcal{R}}\left(L_{m k}^{\mathcal{R}}\right)$ be the profit-to-go function for the manufacturer with initial state $L_{m k}^{\mathcal{R}}$. Then, the recursion can be formulated as follows.

$$
V_{m k}^{\mathcal{R}}\left(L_{m k}^{\mathcal{R}}\right)=w_{k} L_{m k}^{\mathcal{R}}+\max _{\bar{y}_{k}^{\mathcal{R}} \geq \underline{y}_{k}^{\mathcal{R}} \geq L_{m k}^{\mathcal{R}}}\left\{\pi_{m k}^{\mathcal{R}}\left(\bar{y}_{k}^{\mathcal{R}}, \underline{y}_{k}^{\mathcal{R}}\right)+\alpha E V_{m k+1}^{\mathcal{R}}\left[\left(\underline{y}_{k}^{\mathcal{R}}-D_{k}\right)^{+}\right]\right\}
$$

where $V_{m n+1}^{\mathcal{R}}\left(L_{m n+1}^{\mathcal{R}}\right)=w_{n+1} L_{m n+1}^{\mathcal{R}}$ and

$$
\begin{aligned}
\pi_{m k}^{\mathcal{R}}\left(\bar{y}_{k}^{\mathcal{R}}, \underline{y}_{k}^{\mathcal{R}}\right)= & \left(p_{k}-b_{k}\right) E\left(D_{k}\right)-w_{k} \bar{y}_{k}^{\mathcal{R}}-o_{k}\left(\bar{y}_{k}^{\mathcal{R}}-\underline{y}_{k}^{\mathcal{R}}\right)+e_{k} E\left[\min \left(\bar{y}_{k}^{\mathcal{R}}-\underline{y}_{k}^{\mathcal{R}}\right),\left(\bar{y}_{k}^{\mathcal{R}}-D_{k}\right)^{+}\right] \\
& -r_{k} E\left[\left(D_{k}-\bar{y}_{k}^{\mathcal{R}}\right)^{+}\right]-h_{m k} E\left[\left(\underline{y}_{k}^{\mathcal{R}}-D_{k}\right)^{+}\right] .
\end{aligned}
$$


In equation (5.13), the first term is the incomes and the assembly costs from the sales of final products, the second term is the wholesale ordering costs of components, the third terms is the purchasing costs of put options, the fourth term is the incomes from exercising the put options, the fifth term is the urgent ordering costs of components and the last term is the holding costs of components.

Let $V_{m k}^{\mathcal{R}+}\left(L_{m k}^{\mathcal{R}}\right)=V_{m k}^{\mathcal{R}}\left(L_{m k}^{\mathcal{R}}\right)-w_{k} L_{m k}^{\mathcal{R}}$. The recursion can be reformulated as follows.

$$
V_{m k}^{\mathcal{R}+}\left(L_{m k}^{\mathcal{R}}\right)=\max _{\bar{y}_{k}^{\mathcal{R}} \geq \underline{y}_{k}^{\mathcal{R}} \geq L_{m k}^{\mathcal{R}}}\left\{\pi_{m k}^{\mathcal{R}+}\left(\bar{y}_{k}^{\mathcal{R}}, \underline{y}_{k}^{\mathcal{R}}\right)+\alpha E V_{m k+1}^{\mathcal{R}+}\left[\left(\underline{y}_{k}^{\mathcal{R}}-D_{k}\right)^{+}\right]\right\}
$$

where $V_{m n+1}^{\mathcal{R}}\left(L_{m n+1}^{\mathcal{R}}\right)=0$ and

$$
\begin{aligned}
\pi_{m k}^{\mathcal{R}+}\left(\bar{y}_{k}^{\mathcal{R}}, \underline{y}_{k}^{\mathcal{R}}\right)= & \left(p_{k}-b_{k}\right) E\left(D_{k}\right)-w_{k} \bar{y}_{k}^{\mathcal{R}}-o_{k}\left(\bar{y}_{k}^{\mathcal{R}}-\underline{y}_{k}^{\mathcal{R}}\right)+e_{k} E\left[\min \left(\bar{y}_{k}^{\mathcal{R}}-\underline{y}_{k}^{\mathcal{R}}\right),\left(\bar{y}_{k}^{\mathcal{R}}-D_{k}\right)^{+}\right] \\
& -r_{k} E\left[\left(D_{k}-\bar{y}_{k}^{\mathcal{R}}\right)^{+}\right]-h_{m k} E\left[\left(\underline{y}_{k}^{\mathcal{R}}-D_{k}\right)^{+}\right]+\alpha w_{k+1} E\left[\left(\underline{y}_{k}^{\mathcal{R}}-D_{k}\right)^{+}\right] .
\end{aligned}
$$

In equation (5.15), the first six terms are the manufacturer's profit margin in current period and the last term is the discounted value of leftover components at the manufacturer's site in next period. Based on the above analysis, we obtain the following lemma.

Lemma 5.9. For period $k=1, \ldots, n, V_{m k}^{\mathcal{R}+}\left(L_{m k}^{\mathcal{R}}\right)$ is a concave function of $L_{m k}^{\mathcal{R}}$.

This lemma indicates that in the supplier-led model there always exists a unique optimal ordering decision for the manufacturer in each period. As to the specific solution, we have the following proposition.

Proposition 5.10. In the supplier-led model, for period $k=1, \ldots, n$, the optimal component order quantity of the manufacturer is

$$
q_{w k}^{\mathcal{R} *}= \begin{cases}S_{k}^{\mathcal{R} *}-L_{m k}^{\mathcal{R}} & \text { if } L_{m k}^{\mathcal{R}} \leq S_{k}^{\mathcal{R} *} \\ 0 & \text { if } L_{m k}^{\mathcal{R}}>S_{k}^{\mathcal{R} *}\end{cases}
$$

and the optimal put options order quantity of the manufacturer is

$$
q_{m k}^{\mathcal{R} *}= \begin{cases}S_{k}^{\mathcal{R} *}-s_{k}^{\mathcal{R} *} & \text { if } L_{m k}^{\mathcal{R}} \leq s_{k}^{\mathcal{R} *} \\ S_{k}^{\mathcal{R} *}-L_{m k}^{\mathcal{R}} & \text { if } s_{k}^{\mathcal{R} *}<L_{m k}^{\mathcal{R}} \leq S_{k}^{\mathcal{R} *} \\ 0 & \text { if } L_{m k}^{\mathcal{R}}>S_{k}^{\mathcal{R} *}\end{cases}
$$

where $S_{k}^{\mathcal{R} *}=\arg \max _{\bar{y}_{k}^{\mathcal{R}}}\left\{\pi_{m k}^{\mathcal{R}+}\left(\bar{y}_{k}^{\mathcal{R}}, \underline{y}_{k}^{\mathcal{R}}\right)+\alpha E V_{m k+1}^{\mathcal{R}+}\left[\left(\underline{y}_{k}^{\mathcal{R}}-D_{k}\right)^{+}\right]\right\}$

and $s_{k}^{\mathcal{R} *}=\arg \max _{\underline{y}_{k}^{\mathcal{R}}}\left\{\pi_{m k}^{\mathcal{R}+}\left(\bar{y}_{k}^{\mathcal{R}}, \underline{y}_{k}^{\mathcal{R}}\right)+\alpha E V_{m k+1}^{\mathcal{R}+}\left[\left(\underline{y}_{k}^{\mathcal{R}}-D_{k}\right)^{+}\right]\right\}$.

This proposition suggests that in the supplier-led model the optimal ordering policy of the manufacturer in period $k$ depends on the initial state $L_{m k}^{\mathcal{R}}$, the optimal policy $s_{k}^{\mathcal{R} *}$ and the optimal policy $S_{k}^{\mathcal{R} *}$ in period $k$. The manufacturer should guarantee the maximum available quantity of components that satisfies demand to be not less than $S_{k}^{\mathcal{R} *}$ and the minimum available quantity of components that satisfies demand to be not less than $s_{k}^{\mathcal{R} *}$ in period $k$. As to the impact of the contract parameters on the optimal policy $S_{k}^{\mathcal{R} *}$ and the optimal policy $s_{k}^{\mathcal{R} *}$, we get the following corollary.

Corollary 5.11. $S_{k}^{\mathcal{R} *}$ is decreasing in $w_{k}$ and $o_{k}$, but is increasing in $e_{k} . s_{k}^{\mathcal{R} *}$ is constant in $w_{k}$, is increasing in $o_{k}$, but is decreasing in $e_{k}$.

This corollary illustrates that in the supplier-led model the optimal policy $S_{k}^{\mathcal{R} *}$ in period $k$ is negatively affected by the wholesale price and the option price in period $k$, while is positively affected by the exercise price in period $k$. That is, an increase in the wholesale price or the option price in period $k$ may induce an decreased component order quantity for the manufacturer in period $k$. However, an increase in the exercise price in period $k$ may 
induce an increased component order quantity for the manufacturer in period $k$. Moreover, in the supplier-led model the optimal policy $s_{k}^{\mathcal{R} *}$ in period $k$ has nothing to do with the wholesale price in period $k$, while is positively affected by the option price in period $k$ and is negatively affected by the exercise price in period $k$. That is, an increase in the wholesale price or the option price in period $k$ may induce a decreased put options order quantity for the manufacturer in period $k$. However, an increase in the exercise price in period $k$ may induce an increased put options order quantity for the manufacturer in period $k$.

We denote $S_{k}^{\mathcal{R}+}$ as a myopic policy for the manufacturer's component order in period $k$ and $s_{k}^{\mathcal{R}+}$ as a myopic policy for the manufacturer's put options order in period $k$ when the effect of the manufacturer's current ordering decision on her future profit can be ignored. Then, we have the following proposition.

Proposition 5.12. For period $k=1, \ldots, n$, we have $S_{k}^{\mathcal{R}+}=F_{k}^{-1}\left(\frac{r_{k}-w_{k}-o_{k}}{r_{k}-e_{k}}\right)$ and $s_{k}^{\mathcal{R}+}=F_{k}^{-1}\left(\frac{o_{k}}{e_{k}+h_{m k}-\alpha w_{k+1}}\right)$.

This proposition shows that in the supplier-led model the myopic policy $s_{k}^{\mathcal{R}+}$ in period $k$ is not affected by the wholesale price in the period $k$, which is consistent with the result of Corollary 5.11. However, we find that in the supplier-led model the myopic policy $s_{k}^{\mathcal{R}+}$ in period $k$ is positively affected by the wholesale price in period $k+1$. That is, an increase in the wholesale price in period $k+1$ may induce a decreased put options order quantity for the manufacturer in period $k$.

Note that $s_{k}^{\mathcal{R}+}<S_{k}^{\mathcal{R}+}$. This means that in the supplier-led model the key decision for the manufacturer is to ensure that the minimum available quantity of components always falls below the maximum available quantity of components in each period. This inequality equals to $o_{k}<\frac{\left(r_{k}-w_{k}\right)\left(e_{k}+h_{m k}-\alpha w_{k+1}\right)}{r_{k}+h_{m k}-\alpha w_{k+1}}$, which indicates that the option price should be not too high.

\subsection{The supplier's optimal ordering policy}

For period $k=1, \ldots, n$, the supplier produces the components up to satisfying the required component order quantity of the manufacturer, that is, $q_{s k}^{\mathcal{D}}=q_{w k}^{\mathcal{R} *}-L_{s k}^{\mathcal{R}}$. The supplier's expected total profit over all periods is

$$
\begin{aligned}
\Pi_{s}^{\mathcal{R}}\left(\bar{y}_{1}^{\mathcal{R} *}, \ldots, \bar{y}_{n}^{\mathcal{R} *}\right)= & \sum_{k=1}^{n}\left\{w_{k}\left(\bar{y}_{k}^{\mathcal{R} *}-L_{m k}^{\mathcal{R}}\right)+o_{k}\left(\bar{y}_{k}^{\mathcal{R} *}-\underline{y}_{k}^{\mathcal{R} *}\right)-\left(e_{k}+h_{s k}\right) E \min \left[\left(\bar{y}_{k}^{\mathcal{R}}-\underline{y}_{k}^{\mathcal{R}}\right),\left(\bar{y}_{k}^{\mathcal{R}}-D_{k}\right)^{+}\right]\right. \\
& \left.-c_{k}\left(\bar{y}_{k}^{\mathcal{R} *}-L_{m k}^{\mathcal{R}}-L_{s k}^{\mathcal{R}}\right)\right\}
\end{aligned}
$$

Although the supplier adopts the make-to-order production strategy, demand uncertainty is partially transformed from the manufacturer into the supplier.

\section{Discussion}

We present numerical examples to provide important managerial insights into the following fields. First, we illustrate whether two members and the channel can benefit from put option contract or not, when compared to the benchmark model without put option contract. Second, we illustrate which of supply chain structure performs better for two members and the channel. Finally, we illustrate how the demand risk affects the members' decisions and total profits as well as the channel's total profit.

Following Li et al. [14], we mainly focus on a stationary multi-period setting. Partial figures are set as follows: $n=4, \alpha=0.9962, p_{k}=977, b_{k}=72, r_{k}=1200, w_{k}=624, c_{k}=491, h_{s k}=16$ and $h_{m k}=29$. We also assume that demands share the same normal distribution with $\mu_{k}=100$ and $\sigma_{k}=50$. All the values of the parameters satisfy the model assumptions listed in Section 3 and the deduced parameter relationship listed in Section 5.

\subsection{The impact of put option contract}

We compare the models with put option contract against the benchmark model without put option contract under different values of the option and exercise prices to illustrate the impact of put option contract on the members' decisions and total profits as well as the channel's total profit. 
TABLE 1. Comparisons of the manufacturer-led model and the benchmark model.

\begin{tabular}{|c|c|c|c|c|c|c|c|c|c|c|}
\hline \multirow[t]{2}{*}{ Parameter } & \multirow[t]{2}{*}{ Value } & \multicolumn{4}{|c|}{ Benchmark model } & \multicolumn{5}{|c|}{ Manufacturer-led model } \\
\hline & & $S_{k}^{\mathcal{D} *}$ & $\Pi_{s}^{\mathcal{D} *}$ & $\Pi_{m}^{\mathcal{D} *}$ & $\Pi_{c}^{\mathcal{D} *}$ & $S_{k}^{\mathcal{P} *}$ & $s_{k}^{\mathcal{P} *}$ & $\Pi_{s}^{\mathcal{P} *}$ & $\Pi_{m}^{\mathcal{P} *}$ & $\Pi_{c}^{\mathcal{P} *}$ \\
\hline \multirow[t]{7}{*}{$o_{k}$} & 3.8 & 181.45 & 52717 & 100003 & 152720 & 186.14 & 50.81 & 51472 & 105592 & 157065 \\
\hline & 4.8 & 181.45 & 52717 & 100003 & 152720 & 190.30 & 58.87 & 51926 & 105234 & 157160 \\
\hline & 5.8 & 181.45 & 52717 & 100003 & 152720 & 195.20 & 65.99 & 52360 & 104820 & 157180 \\
\hline & 6.8 & 181.45 & 52717 & 100003 & 152720 & 201.23 & 72.47 & 52782 & 104311 & 157094 \\
\hline & 7.8 & 181.45 & 52717 & 100003 & 152720 & 209.23 & 78.52 & 53200 & 103639 & 156838 \\
\hline & 8.8 & 181.45 & 52717 & 100003 & 152720 & 221.72 & 84.27 & 53625 & 102604 & 156229 \\
\hline & 9.8 & 181.45 & 52717 & 100003 & 152720 & 265.68 & 89.82 & 54101 & 99227 & 153328 \\
\hline \multirow{7}{*}{$e_{k}$} & 613 & 181.45 & 52717 & 100003 & 152720 & 265.38 & 78.53 & 53978 & 99590 & 153568 \\
\hline & 614 & 181.45 & 52717 & 100003 & 152720 & 221.46 & 76.36 & 53519 & 102864 & 156384 \\
\hline & 615 & 181.45 & 52717 & 100003 & 152720 & 209.09 & 74.35 & 53135 & 103777 & 156911 \\
\hline & 616 & 181.45 & 52717 & 100003 & 152720 & 201.23 & 72.47 & 52782 & 104311 & 157094 \\
\hline & 617 & 181.45 & 52717 & 100003 & 152720 & 195.35 & 70.71 & 52451 & 104666 & 157117 \\
\hline & 618 & 181.45 & 52717 & 100003 & 152720 & 190.62 & 69.06 & 52136 & 104910 & 157046 \\
\hline & 619 & 181.45 & 52717 & 100003 & 152720 & 186.63 & 67.50 & 51834 & 105076 & 156910 \\
\hline
\end{tabular}

\subsubsection{Manufacturer-led model vs. Benchmark model}

We now study the relationship between the manufacturer-led model and the benchmark model. In the following, we first set $e_{k}=616$ and vary $o_{k}$ from 3.8 to 9.8 , and then set $o_{k}=6.8$ and vary $e_{k}$ from 613 to 619 . The derived results are given in Table 1.

Table 1 shows interesting observations as follows. (1) In the manufacturer-led model, as the option price increases, the optimal policy $S_{k}^{\mathcal{P} *}$ and the optimal policy $s_{k}^{\mathcal{P} *}$ will increase, the supplier's optimal total profit $\Pi_{s}^{\mathcal{P} *}$ will increase, while the manufacturer's optimal total profit $\Pi_{m}^{\mathcal{P} *}$ will decrease. (2) In the manufacturerled model, as the exercise price increases, the optimal policy $S_{k}^{\mathcal{P} *}$ and the optimal policy $s_{k}^{\mathcal{P} *}$ will decrease, the supplier's optimal total profit $\Pi_{s}^{\mathcal{P} *}$ will decrease, while the manufacturer's optimal total profit $\Pi_{m}^{\mathcal{P} *}$ will increase. (3) The optimal policy $S_{k}^{\mathcal{D} *}$ is smaller than the optimal policy $S_{k}^{\mathcal{P} *}$, while is greater than the optimal policy $s_{k}{ }^{*}$. (4) If the option price is low and the exercise price is high, the supplier's optimal total profit is lower in the manufacturer-led model than in the benchmark model. In contrast, if the option price is high and the exercise price is low, the supplier's optimal total profit is higher in the manufacturer-led model than in the benchmark model. (5) If the option price is low and the exercise price is high, the manufacturer's optimal total profit is higher in the manufacturer-led model than in the benchmark model. In contrast, if the option price is high and the exercise price is low, the manufacturer's optimal total profit is lower in the manufacturer-led model than in the benchmark model. (6) The channel's optimal total profit is higher in the manufacturer-led model than in the benchmark model.

Based on the above example experiments, we have the following remark.

Remark 6.1. The relationship between the manufacturer-led model and the benchmark model are related as follows:

(1) $s_{k}^{\mathcal{P} *}<S_{k}^{\mathcal{D} *}<S_{k}^{\mathcal{P} *}$.

(2) $\Pi_{s}^{\mathcal{P} *}<\Pi_{s}^{\mathcal{D} *}$ if $o_{k}$ is low and $e_{k}$ is high; $\Pi_{s}^{\mathcal{P} *}>\Pi_{s}^{\mathcal{D} *}$ if $o_{k}$ is high and $e_{k}$ is low.

(3) $\Pi_{m}^{\mathcal{P} *}>\Pi_{m}^{\mathcal{D} *}$ if $o_{k}$ is low and $e_{k}$ is high; $\Pi_{m}^{\mathcal{P} *}<\Pi_{m}^{\mathcal{D} *}$ if $o_{k}$ is high and $e_{k}$ is low.

(4) $\Pi_{c}^{\mathcal{P} *}>\Pi_{c}^{\mathcal{D} *}$.

Part (1) shows that in terms of the channel's service level, the manufacturer-led model always outperforms than the benchmark model. In terms of the manufacturer's inventory risk, the manufacturer-led model always falls behind than the benchmark model. Parts (2) and (3) show that in terms of the members' total profits, the 


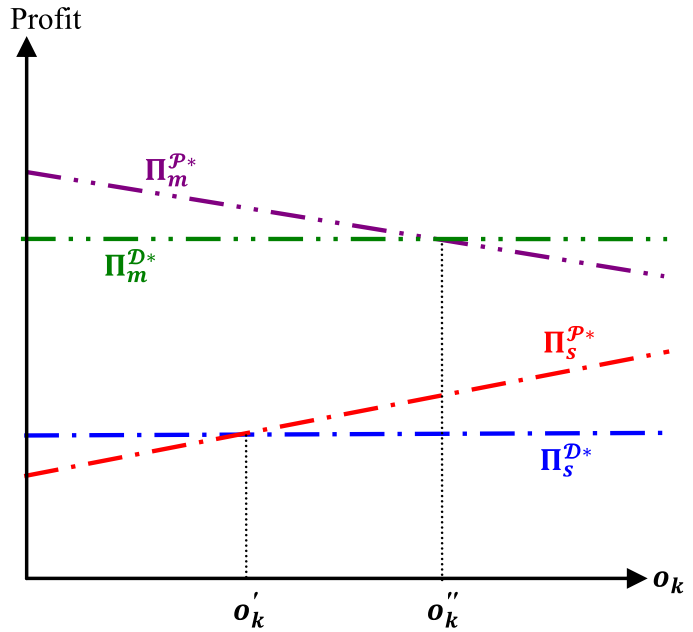

(a)

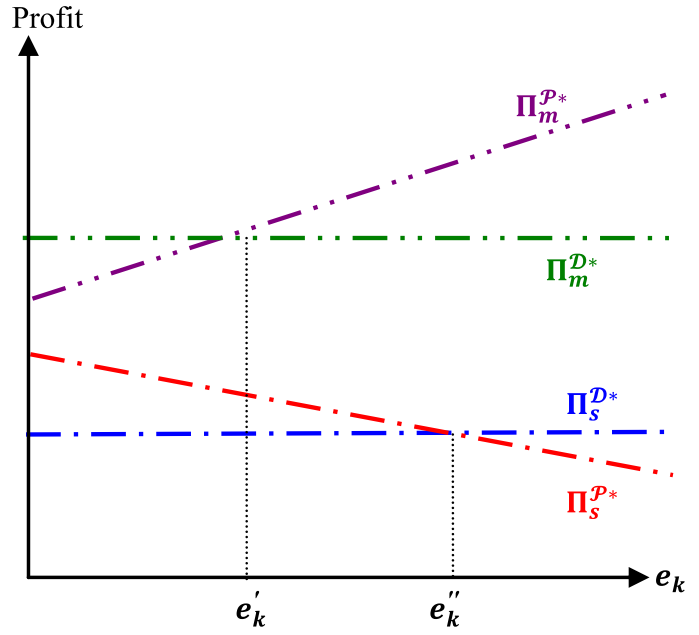

(b)

FIGURE 1. The bargaining range between the leading manufacturer and the followed supplier. (a) The case with a given exercise price. (b) The case with a given option price.

manufacturer-led model cannot always outperform than the benchmark model. Part (4) shows that in terms of the channel's total profit, the manufacturer-led model always outperforms than the benchmark model.

Remark 6.1 suggests that in the manufacturer-led supply chain, put option contract with a low option price and a high exercise price benefits the manufacturer, while put option contract with a high option price and a low exercise price benefits the supplier. Therefore, a bargaining range emerges where two members perform better compared to the case without put option contract. To outline the bargaining range, we depict the following figure.

Figure 1 shows important observations as follows. (1) For a given exercise price, $o_{k}^{\prime}$ and $o_{k}^{\prime \prime}$ exist, which satisfy $\left.\Pi_{s}^{\mathcal{P} *}\right|_{o_{k}=o_{k}^{\prime}}=\Pi_{s}^{\mathcal{D} *}$ and $\left.\Pi_{m}^{\mathcal{P} *}\right|_{o_{k}=o_{k}^{\prime \prime}}=\Pi_{m}^{\mathcal{D} *}$. If $o_{k} \in\left(0, o_{k}^{\prime}\right)$, then $\Pi_{s}^{\mathcal{P} *}<\Pi_{s}^{\mathcal{D} *}$ and $\Pi_{m}^{\mathcal{P} *}>\Pi_{m}^{\mathcal{D} *}$. If $o_{k} \in\left(o_{k}^{\prime}, o_{k}^{\prime \prime}\right)$, then $\Pi_{s}^{\mathcal{P} *}>\Pi_{s}^{\mathcal{D} *}$ and $\Pi_{m}^{\mathcal{P} *}>\Pi_{m}^{\mathcal{D} *}$. If $o_{k} \in\left(o_{k}^{\prime \prime}, \frac{\left(w_{k}-c_{k}\right)\left(e_{k}+h_{m k}-\alpha w_{k+1}\right)}{\left(h_{s k}-\alpha c_{k+1}\right)-\left(h_{m k}-\alpha w_{k+1}\right)}\right)$, then $\Pi_{s}^{\mathcal{P} *}>\Pi_{s}^{\mathcal{D} *}$ and $\Pi_{m}^{\mathcal{P} *}<\Pi_{m}^{\mathcal{D} *}$. Obviously, a win-win situation is achievable by adjusting the option price in the range $\left(o_{k}^{\prime}, o_{k}^{\prime \prime}\right)$. (2) For a given option price, $e_{k}^{\prime}$ and $e_{k}^{\prime \prime}$ exist, which satisfy $\left.\Pi_{m}^{\mathcal{P} *}\right|_{e_{k}=e_{k}^{\prime}}=\Pi_{m}^{\mathcal{D} *}$ and $\left.\Pi_{s}^{\mathcal{P} *}\right|_{e_{k}=e_{k}^{\prime \prime}}=\Pi_{s}^{\mathcal{D} *}$. If $e_{k} \in\left(0, e_{k}^{\prime}\right)$, then $\Pi_{m}^{\mathcal{P} *}<\Pi_{m}^{\mathcal{D} *}$ and $\Pi_{s}^{\mathcal{P} *}>\Pi_{s}^{\mathcal{D} *}$. If $e_{k} \in\left(e_{k}^{\prime}, e_{k}^{\prime \prime}\right)$, then $\Pi_{m}^{\mathcal{P} *}>\Pi_{m}^{\mathcal{D} *}$ and $\Pi_{s}^{\mathcal{P} *}>\Pi_{s}^{\mathcal{D} *}$. If $e_{k} \in\left(e_{k}^{\prime \prime}, \frac{\left[o_{k}-h_{m k}+\alpha w_{k+1}\right]\left[\left(h_{s k}-\alpha c_{k+1}\right)-\left(h_{m k}-\alpha w_{k+1}\right)\right]}{w_{k}-c_{k}}\right)$, then $\Pi_{m}^{\mathcal{P} *}>\Pi_{m}^{\mathcal{D} *}$ and $\Pi_{s}^{\mathcal{P} *}<\Pi_{s}^{\mathcal{D} *}$. Obviously, a win-win situation is achievable by adjusting the exercise price in the range $\left(e_{k}^{\prime}, e_{k}^{\prime \prime}\right)$.

From Remark 6.1, it is realized that in the manufacturer-led supply chain the achievement of the win-win situation is dependent on the put option contract parameters. As a result, the manufacturer, as the leader of the channel, needs to design the contract scientifically to ensure the achievement of the win-win situation so as to guarantee the value of flexibility for put option contract.

\subsubsection{Supplier-led model vs. benchmark model}

We now study the relationship between the supplier-led model and the benchmark model. In the following, we first set $e_{k}=616$ and vary $o_{k}$ from 3.8 to 9.8 , and then set $o_{k}=6.8$ and vary $e_{k}$ from 613 to 619 . The derived results are given in Table 2 .

Table 2 shows interesting observations as follows. (1) In the supplier-led model, as the option price increases, the optimal policy $S_{k}^{\mathcal{R} *}$ will decrease and the optimal policy $s_{k}^{\mathcal{R} *}$ will increase, the supplier's optimal total profit 
TABLE 2. Comparisons of the supplier-led model and the benchmark model.

\begin{tabular}{|c|c|c|c|c|c|c|c|c|c|c|}
\hline \multirow{2}{*}{ Parameter } & \multirow[t]{2}{*}{ Value } & \multicolumn{4}{|c|}{ Benchmark model } & \multicolumn{5}{|c|}{ Supplier-led model } \\
\hline & & $S_{k}^{\mathcal{D} *}$ & $\Pi_{s}^{\mathcal{D} *}$ & $\Pi_{m}^{\mathcal{D} *}$ & $\Pi_{c}^{\mathcal{D} *}$ & $S_{k}^{\mathcal{R} *}$ & $s_{k}^{\mathcal{R} *}$ & $\Pi_{s}^{\mathcal{R} *}$ & $\Pi_{m}^{\mathcal{R} *}$ & $\Pi_{c}^{\mathcal{R} *}$ \\
\hline \multirow[t]{7}{*}{$o_{k}$} & 3.8 & 181.45 & 52717 & 100003 & 152720 & 202.48 & 50.81 & 51359 & 105972 & 157330 \\
\hline & 4.8 & 181.45 & 52717 & 100003 & 152720 & 200.78 & 58.87 & 51883 & 105388 & 157271 \\
\hline & 5.8 & 181.45 & 52717 & 100003 & 152720 & 199.20 & 65.99 & 52355 & 104841 & 157196 \\
\hline & 6.8 & 181.45 & 52717 & 100003 & 152720 & 197.71 & 72.47 & 52779 & 104328 & 157106 \\
\hline & 7.8 & 181.45 & 52717 & 100003 & 152720 & 196.30 & 78.52 & 53157 & 103845 & 157002 \\
\hline & 8.8 & 181.45 & 52717 & 100003 & 152720 & 194.96 & 84.27 & 53493 & 103390 & 15688 \\
\hline & 9.8 & 181.45 & 52717 & 100003 & 152720 & 193.69 & 89.82 & 53787 & 102964 & 156751 \\
\hline \multirow{7}{*}{$e_{k}$} & 613 & 181.45 & 52717 & 100003 & 152720 & 193.80 & 78.53 & 53673 & 103299 & 156972 \\
\hline & 614 & 181.45 & 52717 & 100003 & 152720 & 195.04 & 76.36 & 53392 & 103634 & 157026 \\
\hline & 615 & 181.45 & 52717 & 100003 & 152720 & 196.34 & 74.35 & 53094 & 103977 & 157071 \\
\hline & 616 & 181.45 & 52717 & 100003 & 152720 & 197.71 & 72.47 & 52779 & 104328 & 157106 \\
\hline & 617 & 181.45 & 52717 & 100003 & 152720 & 199.16 & 70.71 & 52446 & 104686 & 157132 \\
\hline & 618 & 181.45 & 52717 & 100003 & 152720 & 200.71 & 69.06 & 52096 & 105051 & 157147 \\
\hline & 619 & 181.45 & 52717 & 100003 & 152720 & 202.37 & 67.50 & 51727 & 105429 & 157156 \\
\hline
\end{tabular}

$\Pi_{s}^{\mathcal{R} *}$ will increase, while the manufacturer's optimal total profit $\Pi_{m}^{\mathcal{R} *}$ will decrease. (2) In the supplier-led model, as the exercise price increases, the optimal policy $S_{k}^{\mathcal{R} *}$ will increase and the optimal policy $s_{k}^{\mathcal{R} *}$ will decrease, the supplier's optimal total profit $\Pi_{s}^{\mathcal{R} *}$ will decrease, while the manufacturer's optimal total profit $\Pi_{m}^{\mathcal{R} *}$ will increase. (3) The optimal policy $S_{k}^{\mathcal{D} *}$ is smaller than the optimal policy $S_{k}^{\mathcal{R} *}$, while is greater than the optimal policy $s_{k}^{\mathcal{R} *}$. (4) If the option price is low and the exercise price is high, the supplier's optimal total profit is lower in the supplier-led model than in the benchmark model. In contrast, if the option price is high and the exercise price is low, the supplier's optimal total profit is higher in the supplier-led model than in the benchmark model.

(5) The manufacturer's optimal total profit is higher in the supplier-led model than in the benchmark model.

(6) The channel's optimal total profit is higher in the supplier-led model than in the benchmark model.

Based on the above example experiments, we have the following remark.

Remark 6.2. The relationship between the supplier-led model and the benchmark model are related as follows:

(1) $s_{k}^{\mathcal{R} *}<S_{k}^{\mathcal{D} *}<S_{k}^{\mathcal{R} *}$.

(2) $\Pi_{s}^{\mathcal{R} *}<\Pi_{s}^{\mathcal{D} *}$ if $o_{k}$ is low and $e_{k}$ is high; $\Pi_{s}^{\mathcal{R} *}>\Pi_{s}^{\mathcal{D} *}$ if $o_{k}$ is high and $e_{k}$ is low.

(3) $\Pi_{m}^{\mathcal{R} *}>\Pi_{m}^{\mathcal{D} *}$.

(4) $\Pi_{c}^{\mathcal{R} *}>\Pi_{c}^{\mathcal{D} *}$

Part (1) shows that in terms of the channel's service level, the supplier-led model always outperforms than the benchmark model. In terms of the manufacturer's inventory risk, the supplier-led model always falls behind than the benchmark model. Parts (2) and (3) show that in terms of the supplier's total profit, the supplier-led model cannot always outperform than the benchmark model. In the terms of the manufacturer's total profit, the supplier-led model always outperforms than the benchmark model. Part (4) shows that in terms of the channel's total profit, the manufacturer-led model outperforms than the benchmark model.

Remark 6.2 suggests that in the supplier-led supply chain, put option contract always benefits the manufacturer, while put option contract with a high option price and a low exercise price benefits the supplier. Hence, a bargaining range emerges where the supplier performs better compared to the case without put option contract. To outline the bargaining range, we depict the following figure.

Figure 2 shows important observations as follows. (1) For a given exercise price, $o_{k}^{\prime}$ exists, which satisfies $\left.\Pi_{s}^{\mathcal{R} *}\right|_{o_{k}=o_{k}^{\prime}}=\Pi_{s}^{\mathcal{D} *}$. If $o_{k} \in\left(0, o_{k}^{\prime}\right)$, then $\Pi_{s}^{\mathcal{R} *}<\Pi_{s}^{\mathcal{D} *}$ and $\Pi_{m}^{\mathcal{R} *}>\Pi_{m}^{\mathcal{D} *}$. If $o_{k} \in\left(o_{k}^{\prime}, \frac{\left(r_{k}-w_{k}\right)\left(e_{k}+h_{m k}-\alpha w_{k+1}\right)}{r_{k}+h_{m k}-\alpha w_{k+1}}\right)$, then 


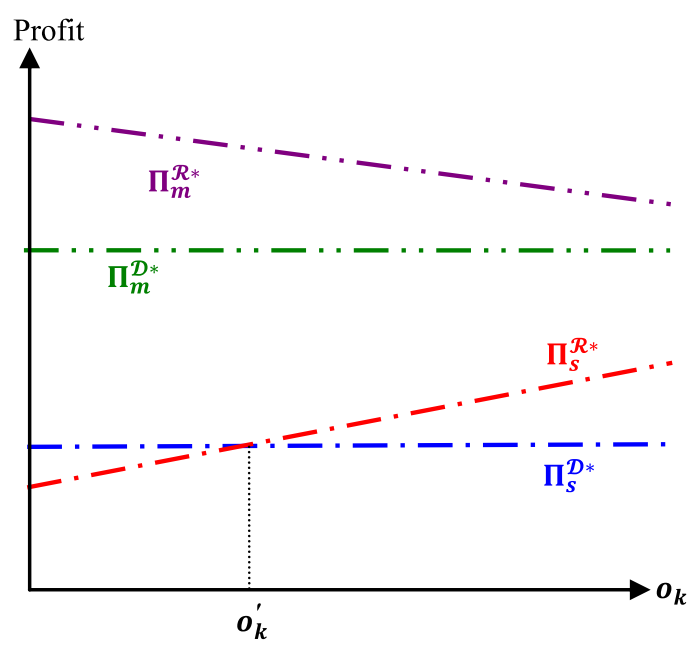

(a)

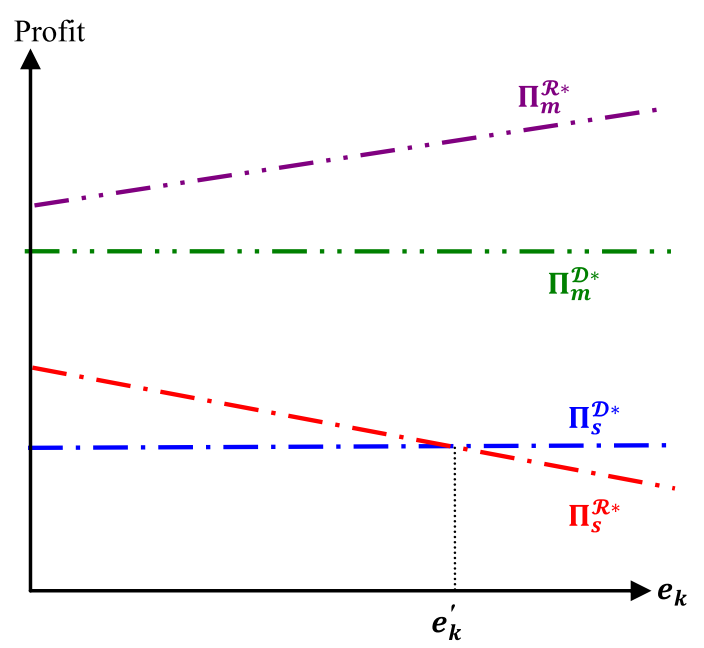

(b)

FiguRE 2. The bargaining range between the leading supplier and the followed manufacturer.

(a) The case with a given exercise price. (b) The case with a given option price.

$\Pi_{s}^{\mathcal{R} *}>\Pi_{s}^{\mathcal{D} *}$ and $\Pi_{m}^{\mathcal{R} *}>\Pi_{m}^{\mathcal{D} *}$. Notably, a win-win situation is achievable by adjusting the option price in the range $\left(o_{k}^{\prime}, \frac{\left(r_{k}-w_{k}\right)\left(e_{k}+h_{m k}-\alpha w_{k+1}\right)}{r_{k}+h_{m k}-\alpha w_{k+1}}\right)$. (2) For a given option price, $e_{k}^{\prime}$ exists, which satisfies $\left.\Pi_{m}^{\mathcal{R} *}\right|_{e_{k}=e_{k}^{\prime}}=\Pi_{m}^{\mathcal{D} *}$. If $e_{k} \in\left(0, e_{k}^{\prime}\right)$, then $\Pi_{s}^{\mathcal{R} *}>\Pi_{s}^{\mathcal{D} *}$ and $\Pi_{m}^{\mathcal{R} *}>\Pi_{m}^{\mathcal{D} *}$. If $e_{k} \in\left(e_{k}^{\prime}, \frac{o_{k}\left(r_{k}+h_{m k}-\alpha w_{k+1}\right)-\left(r_{k}-w_{k}\right)\left(h_{m k}-\alpha w_{k+1}\right)}{r_{k}-w_{k}}\right)$, then $\Pi_{s}^{\mathcal{R} *}<\Pi_{s}^{\mathcal{D} *}$ and $\Pi_{m}^{\mathcal{R} *}>\Pi_{m}^{\mathcal{D} *}$. Notably, a win-win situation is achievable by adjusting the exercise price in the range $\left(0, e_{k}^{\prime}\right)$.

From Remark 6.2, it is realized that in the supplier-led supply chain the assurance of the supplier's profit is dependent on the put option contract parameters. As a result, the supplier, as the leader of the channel, needs to design the contract rationally to ensure the acquisition of his own enough profit so as to guarantee the value of flexibility for put option contract.

\subsection{The impact of supply chain structure}

We compare the manufacturer-led model against the supplier-led model under different values of the option and exercise prices to state the impact of supply chain structure on the members' decisions and total profits as well as the channel's total profit. In the following, we first set $e_{k}=616$ and vary $o_{k}$ from 8.2 to 8.8 , and then set $o_{k}=8.5$ and vary $e_{k}$ from 615.7 to 616.3 . The derived results are given in Table 3 .

Table 3 shows interesting observations as follows. (1) The optimal policy $S_{k}^{\mathcal{P} *}$ may be higher than, equal to, or lower than the optimal policy $S_{k}^{\mathcal{R} *}$. However, the optimal policy $s_{k}^{\mathcal{P} *}$ is always equal to the optimal policy $s_{k}^{\mathcal{R} *}$. (2) The supplier's optimal total profit is always higher in the manufacturer-led model than in the supplierled model. (3) The manufacturer's optimal total profit is always higher in the supplier-led model than in the manufacturer-led model. (4) The channel's total profit is always higher in the supplier-led model than in the manufacturer-led model.

Remark 6.3. The relationship between the manufacturer-led model and the supplier-led model are related as follows:

(1) $s_{k}^{\mathcal{P} *}=s_{k}^{\mathcal{R} *}$.

(2) $\Pi_{s}^{\mathcal{P} *}>\Pi_{s}^{\mathcal{R} *}$. 
TABLE 3. Comparisons of the manufacturer-led model and the supplier-led model.

\begin{tabular}{|c|c|c|c|c|c|c|c|c|c|c|c|}
\hline \multirow[t]{2}{*}{ Parameter } & \multirow[t]{2}{*}{ Value } & \multicolumn{5}{|c|}{ Manufacturer-led model } & \multicolumn{5}{|c|}{ Supplier-led model } \\
\hline & & $S_{k}^{\mathcal{P} *}$ & $s_{k}^{\mathcal{P} *}$ & $\Pi_{s}^{\mathcal{P} *}$ & $\Pi_{m}^{\mathcal{P} *}$ & $\Pi_{\mathcal{C}}^{\mathcal{P} *}$ & $S_{k}^{\mathcal{R} *}$ & $s_{k}^{\mathcal{R} *}$ & $\Pi_{s}^{\mathcal{R} *}$ & $\Pi_{m}^{\mathcal{R} *}$ & $\Pi_{c}^{\mathcal{R} *}$ \\
\hline \multirow[t]{7}{*}{$o_{k}$} & 8.2 & 213.41 & 80.85 & 53368 & 103290 & 156658 & 195.76 & 80.85 & 53297 & 103660 & 156956 \\
\hline & 8.3 & 214.59 & 81.43 & 53410 & 103192 & 156602 & 195.62 & 81.43 & 53331 & 103614 & 156945 \\
\hline & 8.4 & 215.84 & 82.00 & 53453 & 103088 & 156541 & 195.49 & 82.00 & 53364 & 103569 & 156933 \\
\hline & 8.5 & 217.16 & 82.57 & 53495 & 102979 & 156474 & 195.36 & 82.57 & 53397 & 103524 & 156921 \\
\hline & 8.6 & 218.57 & 83.14 & 53538 & 102862 & 156400 & 195.22 & 83.14 & 53430 & 103479 & 156908 \\
\hline & 8.7 & 220.08 & 83.71 & 53581 & 102738 & 156319 & 195.09 & 83.71 & 53462 & 103435 & 156896 \\
\hline & 8.8 & 221.72 & 84.27 & 53625 & 102604 & 156229 & 194.96 & 84.27 & 53493 & 103390 & 156884 \\
\hline \multirow[t]{7}{*}{$\overline{e_{k}}$} & 615.7 & 221.68 & 83.20 & 53611 & 102641 & 156252 & 194.97 & 83.20 & 53480 & 103424 & 156904 \\
\hline & 615.8 & 220.06 & 82.99 & 53572 & 102762 & 156334 & 195.10 & 82.99 & 53453 & 103457 & 156910 \\
\hline & 615.9 & 218.56 & 82.78 & 53533 & 102875 & 156408 & 195.23 & 82.78 & 53425 & 103491 & 156915 \\
\hline & 616.0 & 217.16 & 82.57 & 53495 & 102979 & 156474 & 195.36 & 82.57 & 53397 & 103524 & 156921 \\
\hline & 616.1 & 215.85 & 82.36 & 53458 & 103076 & 156533 & 195.48 & 82.36 & 53369 & 103557 & 156926 \\
\hline & 616.2 & 214.61 & 82.16 & 53421 & 103167 & 156587 & 195.61 & 82.16 & 53341 & 103590 & 156931 \\
\hline & 616.3 & 213.45 & 81.95 & 53384 & 103252 & 156636 & 195.74 & 81.95 & 53312 & 103624 & 156936 \\
\hline
\end{tabular}

TABLE 4. The impact of demand risk.

\begin{tabular}{|c|c|c|c|c|c|c|c|c|c|c|c|c|c|c|}
\hline \multirow[t]{2}{*}{$\sigma_{k}$} & \multicolumn{4}{|c|}{ Benchmark model } & \multicolumn{5}{|c|}{ Manufacturer-led model } & \multicolumn{5}{|c|}{ Supplier-led model } \\
\hline & $S_{k}^{\mathcal{D} *}$ & $\Pi_{s}^{\mathcal{D} *}$ & $\Pi_{m}^{\mathcal{D} *}$ & $\Pi_{c}^{\mathcal{D} *}$ & $S_{k}^{\mathcal{P} *}$ & $s_{k}^{\mathcal{P} *}$ & $\Pi_{\mathcal{S}}^{\mathcal{P} *}$ & $\Pi_{m}^{\mathcal{P} *}$ & $\Pi_{c}^{\mathcal{P} *}$ & $S_{k}^{\mathcal{R} *}$ & $s_{k}^{\mathcal{R} *}$ & $\Pi_{S}^{\mathcal{R} *}$ & $\Pi_{m}^{\mathcal{R} *}$ & $\Pi_{c}^{\mathcal{R} *}$ \\
\hline 35 & 157.02 & 52624 & 102866 & 155489 & 170.86 & 80.73 & 52672 & 105881 & 158553 & 168.40 & 80.73 & 52669 & 105893 & 158562 \\
\hline 40 & 165.16 & 52613 & 101728 & 154341 & 180.98 & 77.98 & 52669 & 105175 & 157843 & 178.17 & 77.98 & 52666 & 105188 & 157853 \\
\hline 45 & 173.31 & 52639 & 100754 & 153394 & 191.10 & 75.22 & 52701 & 104632 & 157333 & 187.94 & 75.22 & 52698 & 104647 & 157344 \\
\hline 50 & 181.45 & 52717 & 100003 & 152720 & 201.23 & 72.47 & 52782 & 104311 & 157094 & 197.71 & 72.47 & 52779 & 104328 & 157106 \\
\hline 55 & 189.60 & 52844 & 99511 & 152355 & 211.35 & 69.72 & 52920 & 104250 & 157170 & 207.48 & 69.72 & 52916 & 104268 & 157184 \\
\hline 60 & 197.74 & 53035 & 99294 & 152329 & 221.47 & 66.96 & 53118 & 104465 & 157582 & 217.25 & 66.96 & 53113 & 104484 & 157597 \\
\hline 65 & 205.89 & 53286 & 99355 & 152642 & 231.59 & 64.21 & 53376 & 104956 & 158332 & 227.02 & 64.21 & 53371 & 104978 & 158349 \\
\hline
\end{tabular}

(3) $\Pi_{m}^{\mathcal{P} *}<\Pi_{m}^{\mathcal{R} *}$.

(4) $\Pi_{c}^{\mathcal{P} *}<\Pi_{c}^{\mathcal{R} *}$.

Part (1) shows that in terms of the manufacturer's inventory risk, the manufacturer-led structure works the same as the supplier-led structure. Parts (2) and (3) show that in terms of the members' total profits, the manufacturer-led structure benefits the supplier and worsens the manufacturer, while the supplier-led structure benefits the manufacturer and worsens the supplier. Part (4) shows that in terms of the channel's total profit, the supplier-led structure outperforms than the manufacturer-led structure.

From Remark 6.3, it is realized that with put option contract the member with more power will obtain lower profit, but the member with less power will obtain higher profit. In addition, with put option contract the power shift from the supplier to the manufacturer will deteriorate the channel efficiency. These above findings provide a reference for the choice of supply chain structure.

\subsection{The impact of demand risk}

We examine the impact of demand risk on the members' decisions and total profits as well as the channel's total profit. As is well known, the demand risk is measured by the standard deviation of demand $(\sigma)$. In the following, we set $o_{k}=6.8, e_{k}=616$ and vary $\sigma$ from 35 to 65 . The derived results are given in Table 4 .

Table 4 shows interesting observations as follows. (1) In the benchmark model, as the demand risk increases, the optimal policy $S_{k}^{\mathcal{D} *}$ will increase. In the manufacturer-led model, as the demand risk increases, the optimal 
policy $S_{k}^{\mathcal{P} *}$ will increase, while the optimal policy $s_{k}^{\mathcal{P} *}$ will decrease. In the supplier-led model, as the demand risk increases, the optimal policy $S_{k}^{\mathcal{R} *}$ will increase, while the optimal policy $s_{k}^{\mathcal{R} *}$ will decrease. (2) In the models of three types, as the demand risk increases, the manufacturer's optimal total profit will first decrease and then increase. (3) In the models of three types, as the demand risk increases, the supplier's optimal total profit will first decrease and then increase. (4) In the models of three types, as the demand risk increases, the channel's optimal total profit will first decrease and then increase.

Based on the above example experiments, we have the following remark.

Remark 6.4. In the models of three types, the optimal total profits of the members and the channel are first decreasing and then increasing in $\sigma_{k}$.

From Remark 6.4, it is realized that high demand risk is not always a bad thing. The key issue for the decisionmakers is to identify the level of demand risk. Therefore, it is advised that the risk observation mechanism must be established so that the decision-makers can keep themselves up-to-date with demand information timely so as to make the decisions rationally based on the dynamic demand information.

\section{Supply ChAin COORDinATION}

We first analyze the integrated model and then explore the explicit condition under which the coordination of the manufacturer-led and supplier-led supply chains can be achieved with put option contract in a multi-period setting. Following Li et al. [14], the multi-period supply chain can be coordinated when the decentralized supply chain performance moves infinitely close to the integrated case.

\subsection{Integrated model}

In the integrated model, the manufacturer and the supplier behavior as an entity to optimize the whole supply chain system performance. For period $k=1, \ldots, n$, when the component production of the integrated supply chain is completed, the maximum available quantity of components to satisfy demand is $\bar{y}_{k}^{\mathcal{J}}=L_{c k}^{\mathcal{J}}+q_{s k}^{\mathcal{J}}$. So, the inventory levels for the integrated supply chain in two successive periods are related as $L_{c k+1}^{\mathcal{J}}=E\left[\left(\bar{y}_{k}^{\mathcal{J}}-D_{k}\right)^{+}\right]$. The expected total profit of the integrated supply chain over all periods is

$$
\Pi_{c}^{\mathcal{J}}\left(\bar{y}_{1}^{\mathcal{J}}, \ldots, \bar{y}_{n}^{\mathcal{J}}\right)=\sum_{k=1}^{n}\left\{\left(p_{k}-b_{k}\right) E\left(D_{k}\right)-c_{k}\left(\bar{y}_{k}^{\mathcal{J}}-L_{c k}^{\mathcal{J}}\right)-r_{k} E\left[\left(D_{k}-\bar{y}_{k}^{\mathcal{J}}\right)^{+}\right]-h_{s k} E\left[\left(\bar{y}_{k}^{\mathcal{J}}-D_{k}\right)^{+}\right]\right\} .
$$

Let $V_{c k}^{\mathcal{J}}\left(L_{c k}^{\mathcal{J}}\right)$ be the profit-to-go function for the integrated supply chain with initial state $L_{c k}^{\mathcal{J}}$. Then, the recursion can be developed as follows.

$$
V_{c k}^{\mathcal{J}}\left(L_{c k}^{\mathcal{J}}\right)=c_{k} L_{c k}^{\mathcal{J}}+\max _{\bar{y}_{k}^{\mathcal{J}} \geq L_{c k}^{\mathcal{J}}}\left\{\pi_{c k}^{\mathcal{J}}\left(\bar{y}_{k}^{\mathcal{J}}\right)+\alpha E V_{c k+1}^{\mathcal{J}}\left[\left(\bar{y}_{k}^{\mathcal{J}}-D_{k}\right)^{+}\right]\right\}
$$

where $V_{c n+1}^{\mathcal{J}}\left(L_{c n+1}^{\mathcal{J}}\right)=c_{n+1} L_{c n+1}^{\mathcal{J}}$ and

$$
\pi_{c k}^{\mathcal{J}}\left(y_{k}^{\mathcal{J}}\right)=\left(p_{k}-b_{k}\right) E\left(D_{k}\right)-c_{k} \bar{y}_{k}^{\mathcal{J}}-r_{k} E\left[\left(D_{k}-\bar{y}_{k}^{\mathcal{J}}\right)^{+}\right]-h_{s k} E\left[\left(\bar{y}_{k}^{\mathcal{J}}-D_{k}\right)^{+}\right] .
$$

In equation (7.3), the first term is the incomes and the assembly costs from the sales of final products, the second term is the production costs, the third term is the urgent ordering costs of components and the last term is the holding costs of components.

Let $V_{c k}^{\mathcal{J}+}\left(L_{c k}^{\mathcal{J}}\right)=V_{c k}^{\mathcal{J}}\left(L_{c k}^{\mathcal{J}}\right)-c_{k} L_{c k}^{\mathcal{J}}$. The recursion can be reformulated as follows.

$$
V_{c k}^{\mathcal{J}+}\left(L_{c k}^{\mathcal{J}}\right)=\max _{\bar{y}_{k}^{\mathcal{J}} \geq L_{c k}^{\mathcal{J}}}\left\{\pi_{c k}^{\mathcal{J}+}\left(\bar{y}_{k}^{\mathcal{J}}\right)+\alpha E V_{k+1}^{\mathcal{J}+}\left[\left(\bar{y}_{k}^{\mathcal{J}}-D_{k}\right)^{+}\right]\right\}
$$


where $V_{c n+1}^{\mathcal{J}+}\left(L_{c n+1}^{\mathcal{J}}\right)=0$ and

$$
\pi_{c k}^{\mathcal{J}+}\left(y_{k}^{\mathcal{J}}\right)=\left(p_{k}-b_{k}\right) E\left(D_{k}\right)-c_{k} \bar{y}_{k}^{\mathcal{J}}-r_{k} E\left[\left(D_{k}-\bar{y}_{k}^{\mathcal{J}}\right)^{+}\right]-h_{s k} E\left[\left(\bar{y}_{k}^{\mathcal{J}}-D_{k}\right)^{+}\right]+\alpha c_{k+1} E\left[\left(\bar{y}_{k}^{\mathcal{J}}-D_{k}\right)^{+}\right] .
$$

In equation (7.5), the first four terms are the supply chain's profit margin in current period and the last term is the discounted value of leftover components at the supply chain's site in next period. Based on the above analysis, we obtain the following lemma.

Lemma 7.1. For period $k=1, \ldots, n, V_{c k}^{\mathcal{J}+}\left(L_{c k}^{\mathcal{J}}\right)$ is a concave function of $L_{c k}^{\mathcal{J}}$.

This lemma indicates that in the integrated model there always exists a unique optimal production decision for the integrated supply chain in each period. As to the specific solution, we have the following proposition.

Proposition 7.2. In the integrated model, for period $k=1, \ldots, n$, the optimal production quantity for the integrated supply chain is

$$
q_{s k}^{\mathcal{J} *}= \begin{cases}S_{k}^{\mathcal{J} *}-L_{c k}^{\mathcal{J}} & \text { if } L_{c k}^{\mathcal{J}} \leq S_{k}^{\mathcal{J} *} \\ 0 & \text { if } L_{c k}^{\mathcal{J}}>S_{k}^{\mathcal{J} *}\end{cases}
$$

where $S_{k}^{\mathcal{J} *}=\arg \max _{\bar{y}_{k}^{\mathcal{J}}}\left\{\pi_{c k}^{\mathcal{J}+}\left(\bar{y}_{k}^{\mathcal{J}}\right)+\alpha E V_{c k+1}^{\mathcal{J}+}\left[\left(\bar{y}_{k}^{\mathcal{J}}-D_{k}\right)^{+}\right]\right\}$

This proposition shows that in the integrated model the optimal production policy for the integrated supply chain in period $k$ depends on the initial state $L_{c k}^{\mathcal{J}}$ and the optimal policy $S_{k}^{\mathcal{J} *}$ in period $k$. The integrated supply chain should guarantee the maximum available quantity of components that satisfies demand to be not less than $S_{k}^{\mathcal{J} *}$ in period $k$. As to the impact of the production cost on the optimal policy $S_{k}^{\mathcal{J} *}$, we get the following corollary.

Corollary 7.3. For period $k=1, \ldots, n, S_{k}^{\mathcal{J} *}$ is decreasing in $c_{k}$.

This corollary illustrates that in the integrated model the optimal policy $S_{k}^{\mathcal{J} *}$ in period $k$ is negatively affected by the production cost in period $k$. That is, an increase in the production cost in period $k$ may induce a decreased production quantity for the integrated supply chain in period $k$.

We denote $S_{k}^{\mathcal{J}+}$ as a myopic policy for the integrated supply chain's production when the impact of the system's current production decision on the future profit can be ignored. Then, we have the following proposition.

Proposition 7.4. For period $k=1, \ldots, n$, we have $S_{k}^{\mathcal{J}+}=F_{k}^{-1}\left(\frac{r_{k}-c_{k}}{r_{k}+h_{s k}-\alpha c_{k+1}}\right)$.

This proposition shows that in the integrated model the myopic policy $S_{k}^{\mathcal{J}+}$ in period $k$ is related to the urgent order cost and the holding cost in period $k$, while is unrelated to the retail price and the assemble cost in period $k$. Moreover, in the integrated model the myopic policy $S_{k}^{\mathcal{J}+}$ in period $k$ is negatively affected by the production cost in period $k$, which is in accordance with the result of Corollary 7.3. However, we find that in the integrated model the myopic policy $S_{k}^{\mathcal{J}+}$ in period $k$ is positively affected by the production cost in period $k+1$. That is, an increase in the production cost in period $k+1$ may generate an increased production quantity for the integrated supply chain in period $k$.

\subsection{Manufacturer-led supply chain coordination}

In a manufacturer-led supply chain, it is sufficient for the manufacturer to design the contract under which the supplier is stimulated to produce nearly the same quantity of components as the production quantity of the integrated supply chain to achieve the channel coordination in a multi-period setting. Then, we have the following proposition.

Proposition 7.5. The manufacturer-led supply chain is coordinated through put option contract when

$$
e_{k}=\frac{\left(w_{k}+o_{k}\right)\left(r_{k}+h_{s k}-\alpha c_{k+1}\right)-r_{k}\left(c_{k}+h_{s k}-\alpha c_{k+1}\right)}{r_{k}-c_{k}} .
$$

This proposition indicates that the coordination condition is related to the urgent ordering cost and the holding cost, but is unrelated to the retail price and the assemble cost. However, If insufficient components are 
TABLE 5. The coordination of the manufacturer-led supply chain.

\begin{tabular}{|c|c|c|c|c|c|c|c|c|c|}
\hline \multirow[t]{2}{*}{$o_{k}$} & \multicolumn{4}{|c|}{ Benchmark model without coordination } & \multicolumn{5}{|c|}{ Manufacturer-led model with coordination } \\
\hline & $S_{k}^{\mathcal{D} *}$ & $\Pi_{s}^{\mathcal{D} *}$ & $\Pi_{m}^{\mathcal{D} *}$ & $\Pi_{c}^{\mathcal{D} *}$ & $S_{k}^{\mathcal{P *}}$ & $s_{k}^{\mathcal{P} *}$ & $\Pi_{s}^{\mathcal{P} *}$ & $\Pi_{m}^{\mathcal{P} *}$ & $\Pi_{c}^{\mathcal{P} *}$ \\
\hline 8.2 & 181.45 & 52717 & 100003 & 152720 & 198.36 & 77.25 & 52722 & 104309 & 157031 \\
\hline 8.3 & 181.45 & 52717 & 100003 & 152720 & 198.36 & 77.62 & 52728 & 104297 & 157025 \\
\hline 8.4 & 181.45 & 52717 & 100003 & 152720 & 198.36 & 77.98 & 52734 & 104285 & 157019 \\
\hline 8.5 & 181.45 & 52717 & 100003 & 152720 & 198.36 & 78.34 & 52739 & 104273 & 157013 \\
\hline 8.6 & 181.45 & 52717 & 100003 & 152720 & 198.36 & 78.69 & 52745 & 104261 & 157006 \\
\hline 8.7 & 181.45 & 52717 & 100003 & 152720 & 198.36 & 79.04 & 52751 & 104250 & 157000 \\
\hline 8.8 & 181.45 & 52717 & 100003 & 152720 & 198.36 & 79.38 & 52756 & 104238 & 156995 \\
\hline
\end{tabular}

backordered, the coordination condition is dependent on the retail price and the assemble cost [8]. In addition, the coordination condition is unrelated to the demand distribution. This feature makes the application of the coordination condition easy.

The coordination condition mentioned above is a unilateral solution from the perspective of the supplier. In the following, we explore how the manufacturer decides the ordering policy to achieve the bilateral coordination. Then, we have the following proposition.

Proposition 7.6. When the manufacturer-led supply chain is coordinated bilaterally, the manufacturer's myopic policy is

(1) If $o_{k}<\frac{\left(r_{k}-c_{k}\right)\left[\left(h_{s k}-\alpha c_{k+1}\right)-\left(h_{m k}-\alpha w_{k+1}\right)\right]-\left(w_{k}-c_{k}\right)\left(r_{k}+h_{s k}-\alpha c_{k+1}\right)}{c_{k}+h_{s k}-\alpha c_{k+1}}$, we have $s_{k}^{\mathcal{P}+}=0$;

(2) If $\frac{\left(r_{k}-c_{k}\right)\left[\left(h_{s k}-\alpha c_{k+1}\right)-\left(h_{m k}-\alpha w_{k+1}\right)\right]-\left(w_{k}-c_{k}\right)\left(r_{k}+h_{s k}-\alpha c_{k+1}\right)}{c_{k}+h_{s k}-\alpha c_{k+1}}<o_{k}<\frac{\left(w_{k}-c_{k}\right)\left(e_{k}+h_{m k}-\alpha w_{k+1}\right)}{\left(h_{s k}-\alpha c_{k+1}\right)-\left(h_{m k}-\alpha w_{k+1}\right)}$,

we have $s_{k}^{\mathcal{P}+}=F_{k}^{-1}\left(\frac{o_{k}\left(r_{k}-c_{k}\right)}{\left(w_{k}+o_{k}-c_{k}\right)\left(r_{k}+h_{s k}-\alpha c_{k+1}\right)-\left(r_{k}-c_{k}\right)\left[\left(h_{s k}-\alpha c_{k+1}\right)-\left(h_{m k}-\alpha w_{k+1}\right)\right]}\right)$.

This proposition shows the breaking point below which the manufacturer can send back all the leftover components to the supplier by exercising the put options so that the supplier's inventory risk is relatively high. However, when the option price is above the breaking point, the manufacturer can send back part of leftover components to the supplier by exercising the put options so that the supplier's inventory risk is relatively low.

In the following, we give a numerical illustration of the manufacturer-led supply chain coordination. Similar to Section 6, we also focus on a stationary multi-period setting. Partial figures are set as follows: $n=4, \alpha=0.9962$, $p_{k}=977, b_{k}=72, r_{k}=1200, w_{k}=624, c_{k}=491, h_{s k}=16, h_{m k}=29$ and $D_{k} \sim N(100,50)$.

Tables 3 and 5 show that in the manufacturer-led supply chain, the channel's total profit is higher with coordination than without it. This means that additional profits will generate from the coordination. In addition, with a coordinating contract, both the followed supplier and the dominated manufacturer are better off compared to the case without put option contract.

\subsection{Supplier-led supply chain coordination}

In a supplier-led supply chain, it is sufficient for the supplier to design the contract under which the manufacturer is stimulated to purchase nearly the same quantity of components as the production quantity of the integrated supply chain to achieve the channel coordination in a multi-period setting. Then, we have the following proposition.

Proposition 7.7. The supplier-led supply chain is coordinated through put option contract when

$$
e_{k}=\frac{\left(w_{k}+o_{k}\right)\left(r_{k}+h_{s k}-\alpha c_{k+1}\right)-r_{k}\left(c_{k}+h_{s k}-\alpha c_{k+1}\right)}{r_{k}-c_{k}} .
$$

By comparing Proposition 7.7 against Proposition 7.5, we easily find that the coordination condition of the manufacturer-led supply chain is the same as that of the supplier-led supply chain. The key distinction is that the 
TABLE 6 . The coordination of the supplier-led supply chain.

\begin{tabular}{|c|c|c|c|c|c|c|c|c|c|}
\hline \multirow[t]{2}{*}{$o_{k}$} & \multicolumn{4}{|c|}{ Benchmark model without coordination } & \multicolumn{5}{|c|}{ Supplier-led model with coordination } \\
\hline & $S_{k}^{\mathcal{D} *}$ & $\Pi_{s}^{\mathcal{D} *}$ & $\Pi_{m}^{\mathcal{D} *}$ & $\Pi_{c}^{\mathcal{D} *}$ & $S_{k}^{\mathcal{R} *}$ & $s_{k}^{\mathcal{R} *}$ & $\Pi_{s}^{\mathcal{R} *}$ & $\Pi_{m}^{\mathcal{R} *}$ & $\Pi_{c}^{\mathcal{R} *}$ \\
\hline 8.2 & 181.45 & 52717 & 100003 & 152720 & 198.36 & 77.25 & 52722 & 104309 & 157031 \\
\hline 8.3 & 181.45 & 52717 & 100003 & 152720 & 198.36 & 77.62 & 52728 & 104297 & 157025 \\
\hline 8.4 & 181.45 & 52717 & 100003 & 152720 & 198.36 & 77.98 & 52734 & 104285 & 157019 \\
\hline 8.5 & 181.45 & 52717 & 100003 & 152720 & 198.36 & 77.34 & 52739 & 104273 & 157013 \\
\hline 8.6 & 181.45 & 52717 & 100003 & 152720 & 198.36 & 78.69 & 52745 & 104261 & 157006 \\
\hline 8.7 & 181.45 & 52717 & 100003 & 152720 & 198.36 & 79.03 & 52751 & 104250 & 157000 \\
\hline 8.8 & 181.45 & 52717 & 100003 & 152720 & 198.36 & 79.38 & 52756 & 104238 & 156994 \\
\hline
\end{tabular}

supplier's production and the manufacturer's ordering need be coordinated simultaneously in the manufacturerled supply chain. However, only the manufacturer's ordering need be coordinated in the supplier-led supply chain.

In the following, we give a numerical illustration of the supplier-led supply chain coordination. Similar to Section 6, we also focus on a stationary multi-period setting. Partial figures are set as follows: $n=4, \alpha=0.9962$, $p_{k}=977, b_{k}=72, r_{k}=1200, w_{k}=624, c_{k}=491, h_{s k}=16, h_{m k}=29$ and $D_{k} \sim N(100,50)$.

Tables 3 and 6 show in the supplier-led supply chain, the channel's total profit is higher with coordination than without it. This implies that additional profits will generate from the coordination. In addition, with a coordinating contract, both the dominated supplier and the followed manufacturer are better off compared to the case without put option contract.

\section{Conclusions And suggestions}

We develop a model framework to incorporate put option contract and supply chain structures into a supply chain with a supplier and a manufacturer in a multi-period setting. We study the multi-period production for the supplier and the multi-period purchasing for the manufacturer with put option contract under two supply chain structures. We apply the method of dynamic programming to characterize the structures of optimal policies and provide a myopic approach to evaluate the myopic values of optimal policies. By benchmarking the model without put option contract as the evaluation criterion, we explore the impact of put option contract on the members' decisions and total profits as well as the channel's total profit. By comparing the models with two power structures, we discuss the impact of supply chain structure on the members' decisions and total profits as well as the channel's total profit. We study the impact of demand risk on the members' decisions and total profits as well as the channel's total profit. Finally, we derive the explicit condition for achieving the coordination of the multi-period supply chain through put option contract under two supply chain structures.

This paper reveals interesting results as follows. (1) In the manufacturer-led structure, put option contract always benefits the channel, while benefits the manufacturer under a low option price and a high exercise price and benefits the supplier under a high option price and a low exercise price. (2) In the supplier-led structure, put option contract always benefits the channel and the manufacturer, while benefits the supplier under a high option price and a low exercise price. (3) The manufacturer-led structure is more profitable for the supplier than the supplier-led structure. The supplier-led structure is more profitable for the manufacturer and the channel than the manufacturer-led structure. (4) Under two supply chain structures, the optimal total profits of the members and the channel are first decreasing and then increasing in the demand risk. (5) The manufacturer-led supply chain can be coordinated bilaterally through put option contract, while the supplier-led supply chain can be coordinated unilaterally through put option contract.

This paper provides managerial implications as follows. (1) In the manufacturer-led structure, whether two members benefit from put option contract depends on the values of the option and exercise prices. Hence, 
a bargaining range emerges where two members perform better compared to the case without put option contract. As a result, the manufacture, as the leader of the supply chain, needs to design the contract properly to reach a win-win situation. (2) In the supplier-led structure, the manufacturer always benefits from put option contract. However, whether the supplier benefits from put option contract depends on the values of the option and exercise prices. Hence, a bargaining range emerges where the supplier performs better compared to the case without put option contract. As a result, the supplier, as the leader of the supply chain, needs to design the contract scientifically to guarantee his own profit. (3) With put option contract, the member with more power obtains lower profit, but the member with less power obtains higher profit. In addition, the power shift from the supplier to the manufacturer deteriorates the channel efficiency in the presence of put option contract. As a result, a reference on how to choose the appropriate supply chain structure has been proposed. (4) High demand risk is not always a bad thing for two members and the channel. As a result, the risk observation mechanism is advised to be established to help the decision-makers keep updating demand information and make the decisions dynamically.

This paper has several limitations and can be extended in the future study. For example, this paper mainly focuses on one supply chain with one supplier and one manufacturer. In the future, it can be extended to examine one supply chain consisting of multiple suppliers and one manufacturer. Another limitation is that the retail price is assumed to be exogenous and is irrelevant with the demand. It is interesting to examine the optimal joint ordering and pricing policy with price-dependent demand. Lastly, the supplier and the manufacturer are assumed to be risk-neutral. Another possible extension is to integrate the members' risk preferences into the model.

\section{Appendix A.}

Proof of Lemma 4.1. From equation (4.5), it is easy to derive that $\pi_{m k}^{\mathcal{D}+}\left(\bar{y}_{k}^{\mathcal{D}}\right)$ is concave in $\bar{y}_{k}^{\mathcal{D}}$ for all periods. For period $k=n$, because $V_{m n+1}^{\mathcal{D}+}\left(L_{m n+1}^{\mathcal{D}}\right)=0, \pi_{m n}^{\mathcal{D}+}\left(\bar{y}_{n}^{\mathcal{D}}\right)$ is the sole component of $V_{m n}^{\mathcal{D}+}\left(L_{m n}^{\mathcal{D}}\right)$. Therefore, $V_{m n}^{\mathcal{D}+}\left(L_{m n}^{\mathcal{D}}\right)$ is concave in $\bar{y}_{n}^{\mathcal{D}}$ and thus is concave in $L_{m n}^{\mathcal{D}}$. For period $k=1, \ldots, n-1$, because $E V_{m k+1}^{\mathcal{D}+}\left[\left(\bar{y}_{k}^{\mathcal{D}}-D_{k}\right)^{+}\right]$is concave, $V_{m k}^{\mathcal{D}+}\left(L_{m k}^{\mathcal{D}}\right)$ is consisted of two concave functions. Therefore, $V_{m k}^{\mathcal{D}+}\left(L_{m k}^{\mathcal{D}}\right)$ is concave in $\bar{y}_{k}^{\mathcal{D}}$ and thus is concave in $L_{m k}^{\mathcal{D}}$.

Proof of Proposition 4.2. In the benchmark model, for period $k=1, \ldots, n$, the optimal solution for the decision variable $\bar{y}_{k}^{\mathcal{D}}$ is characterized by $\bar{y}_{k}^{\mathcal{D} *}=\left\{\begin{array}{ll}S_{k}^{\mathcal{D} *} & \text { if } L_{m k}^{\mathcal{D}} \leq S_{k}^{\mathcal{D} *} \\ L_{m k}^{\mathcal{D}} & \text { if } L_{m k}^{\mathcal{D}}>S_{k}^{\mathcal{D} *},\end{array}\right.$ where $S_{k}^{\mathcal{D} *}=\arg \max _{\bar{y}_{k}^{\mathcal{D}}}\left\{\pi_{m k}^{\mathcal{D}+}\left(\bar{y}_{k}^{\mathcal{D}}\right)+\alpha E V_{m k+1}^{\mathcal{D}+}\left[\left(\bar{y}_{k}^{\mathcal{D}}-D_{k}\right)^{+}\right]\right\}$. Since $q_{w k}^{\mathcal{D} *}=\bar{y}_{k}^{\mathcal{D} *}-L_{m k}^{\mathcal{D}}$, we can derive the above result.

Proof of Corollary 4.3. Let $U_{m k}^{\mathcal{D}+}\left(\bar{y}_{k}^{\mathcal{D}}\right)=\pi_{m k}^{\mathcal{D}+}\left(\bar{y}_{k}^{\mathcal{D}}\right)+\alpha E V_{m k+1}^{\mathcal{D}+}\left[\left(\bar{y}_{k}^{\mathcal{D}}-D_{k}\right)^{+}\right]$. It is easy to derive that $\frac{\partial U_{m k}^{\mathcal{D}+}\left(\bar{y}_{k}^{\mathcal{D}}\right)}{\partial \bar{y}_{k}^{D} \partial w_{k}}<0$, which means that $S_{k}^{\mathcal{D} *}$ is a decreasing function of $w_{k}$.

Proof of Proposition 4.4. From equation (4.5), we have $\frac{d \pi_{m k}^{\mathcal{D}+}\left(\bar{y}_{k}^{\mathcal{D}}\right)}{d y_{k}^{\mathcal{D}}}=\left(r_{k}-w_{k}\right)-\left(r_{k}+h_{m k}-\alpha w_{k+1}\right) F_{k}\left(\bar{y}_{k}^{\mathcal{D}}\right)$ and $\frac{d^{2} \pi_{m k}^{\mathcal{D}+}\left(\bar{y}_{k}^{\mathcal{D}}\right)}{d\left(\bar{y}_{k}^{\mathcal{D}}\right)}=-\left(r_{k}+h_{m k}-\alpha w_{k+1}\right) f_{k}\left(\bar{y}_{k}^{\mathcal{D}}\right)<0$. Thus, we conclude that there is an optimal value for the decision variable $\bar{y}_{k}^{\mathcal{D}}$ to maximize $\pi_{m k}^{\mathcal{D}+}\left(\bar{y}_{k}^{\mathcal{D}}\right)$. Let $\frac{d \pi_{m k}^{\mathcal{D}+}\left(\bar{y}_{k}^{\mathcal{D}}\right)}{d y_{k}^{\mathcal{D}}}=0$, we can derive the above result.

Proof of Lemma 5.1. The proof process of Lemma 5.1 is similar with that of Lemma 4.1 and thus is omitted. 
Proof of Proposition 5.2. In the manufacturer-led model, for period $k=1, \ldots, n$, the optimal solution for the decision variable $\bar{y}_{k}^{\mathcal{P}}$ is given by $\bar{y}_{k}^{\mathcal{P} *}= \begin{cases}S_{k}^{\mathcal{P} *} & \text { if } \underline{y}_{k}^{\mathcal{P}} \leq S_{k}^{\mathcal{P} *} \\ \underline{y}_{k}^{\mathcal{P}} & \text { if } \underline{y}_{k}>S_{k}^{\mathcal{P} *}, \quad \text { where }\end{cases}$ $S_{k}^{\mathcal{P} *}=\arg \max _{\bar{y}_{k}^{\mathcal{P}}}\left\{\pi_{s k}^{\mathcal{P}+}\left(\bar{y}_{k}^{\mathcal{P}}\right)+\alpha E V_{s k+1}^{\mathcal{P}+}\left(\min \left[\left(\bar{y}_{k}^{\mathcal{P}}-\underline{y}_{k}^{\mathcal{P}}\right),\left(\bar{y}_{k}^{\mathcal{P}}-D_{k}\right)^{+}\right]\right)\right\}$.

Since $q_{s k}^{\mathcal{P} *}=\bar{y}_{k}^{\mathcal{P} *}-L_{m k}^{\mathcal{P}}-L_{s k}^{\mathcal{P}}$, we can derive the above result.

Proof of Corollary 5.3. Let $U_{s k}^{\mathcal{P}+}\left(\bar{y}_{k}^{\mathcal{P}}\right)=\pi_{s k}^{\mathcal{P}+}\left(\bar{y}_{k}^{\mathcal{P}}\right)+\alpha E V_{s k+1}^{\mathcal{P}+}\left(\min \left[\left(\bar{y}_{k}^{\mathcal{P}}-\underline{y}_{k}^{\mathcal{P}}\right),\left(\bar{y}_{k}^{\mathcal{P}}-D_{k}\right)^{+}\right]\right)$. It is easy to see that $\frac{\partial^{2} U_{s k}^{\mathcal{P}+}\left(\bar{y}_{k}^{\mathcal{P}}\right)}{\partial \bar{y}_{k}^{\mathcal{P}} \partial w_{k}}>0, \frac{\partial^{2} U_{s k}^{\mathcal{P}+}\left(\bar{y}_{k}^{\mathcal{P}}\right)}{\partial \bar{y}_{k}^{\mathcal{P}} \partial o_{k}}>0$ and $\frac{\partial^{2} U_{s k}^{\mathcal{P}+}\left(\bar{y}_{k}^{\mathcal{P}}\right)}{\partial \bar{y}_{k}^{\mathcal{P}} \partial e_{k}}<0$, which means that $S_{k}^{\mathcal{P} *}$ is an increasing function of $w_{k}$ and $o_{k}$, but is a decreasing function of $e_{k}$.

Proof of Proposition 5.4. From equation (5.5), we derive $\frac{d \pi_{s k}^{\mathcal{P}+}\left(\bar{y}_{k}^{\mathcal{P}}\right)}{d y_{k}^{\mathcal{P}}}=\left(w_{k}+o_{k}-c_{k}\right)-\left(e_{k}+h_{s k}-\alpha c_{k+1}\right)$ $F_{k}\left(\bar{y}_{k}^{\mathcal{P}}\right)$ and $\frac{d^{2} \pi_{s k}^{\mathcal{P}+}\left(\bar{y}_{k}^{\mathcal{P}}\right)}{d\left(\bar{y}_{k}^{\mathcal{P}}\right)}=-\left(e_{k}+h_{s k}-\alpha c_{k+1}\right) f_{k}\left(\bar{y}_{k}^{\mathcal{P}}\right)<0$. Thus, we conclude that there is an optimal value for the decision variable $\bar{y}_{k}^{\mathcal{P}}$ to maximize $\pi_{s k}^{\mathcal{P}+}\left(\bar{y}_{k}^{\mathcal{P}}\right)$. Let $\frac{d \pi_{s k}^{\mathcal{P}+}\left(\bar{y}_{k}^{\mathcal{P}}\right)}{d y_{k}^{\mathcal{P}}}=0$, we can derive the above result.

Proof of Lemma 5.5. The proof process of Lemma 5.5 is similar with that of Lemma 4.1 and thus is omitted.

Proof of Proposition 5.6. In the manufacturer-led model, for period $k=1, \ldots, n$, the optimal solution for the decision variable $\underline{y}_{k}^{\mathcal{P}}$ is given by $\underline{y}_{k}^{\mathcal{P} *}=\left\{\begin{array}{ll}s_{k}^{\mathcal{P}^{*}} & \text { if } L_{m k}^{\mathcal{P}} \leq s_{k}^{\mathcal{P} *} \\ L_{m k}^{\mathcal{P}} & \text { if } L_{m k}^{\mathcal{P}}>s_{k}^{\mathcal{P} *}\end{array}\right.$, where $s_{k}^{\mathcal{P} *}=\arg \max _{\underline{y}_{k}^{\mathcal{P}}}\left\{\pi_{m k}^{\mathcal{P}+}\left(\underline{y}_{k}^{\mathcal{P}}\right)+\alpha E V_{m k+1}^{\mathcal{P}+}\left[\left(\underline{y}_{k}^{\mathcal{P}}-D_{k}\right)^{+}\right]\right\}$.

Since $q_{m k}^{\mathcal{P} *}=\bar{y}_{k}^{\mathcal{P} *}-\underline{y}_{k}^{\mathcal{P} *}$, we can derive the above result.

Proof of Corollary 5.7. Let $U_{m k}^{\mathcal{P}+}\left(\underline{y}_{k}^{\mathcal{P}}\right)=\pi_{m k}^{\mathcal{P}+}\left(\underline{y}_{k}^{\mathcal{P}}\right)+\alpha E V_{m k+1}^{\mathcal{P}+}\left[\left(\underline{y}_{k}^{\mathcal{P}}-D_{k}\right)^{+}\right]$. It is easy to see that $\frac{\partial^{2} U_{m k}^{\mathcal{P}+}\left(\underline{y}_{k}^{\mathcal{P}}\right)}{\partial \underline{y}_{k}^{\mathcal{P}} \partial w_{k}}=0, \frac{\partial^{2} U_{m k}^{\mathcal{P}}\left(\underline{y}_{k}^{\mathcal{P}}\right)}{\partial \underline{y}_{k}^{\mathcal{P} \partial \partial o_{k}}}>0$ and $\frac{\partial^{2} U_{m k}^{\mathcal{P}+}\left(\underline{y}_{k}^{\mathcal{P}}\right)}{\partial \underline{y}_{k}^{\mathcal{P}} \partial e_{k}}<0$, which means that $s_{k}^{\mathcal{P} *}$ is a constant function of $w_{k}$, is an increasing function of $o_{k}$, but is a decreasing function of $e_{k}$.

Proof of Proposition 5.8. From equation (5.10), we derive $\frac{d \pi_{m k}^{\mathcal{P}+}\left(\underline{y}_{k}^{\mathcal{P}}\right)}{d y_{k}^{\mathcal{P}}}=o_{k}-\left(e_{k}+h_{m k}-\alpha w_{k+1}\right) F_{k}\left(\underline{y}_{k}^{\mathcal{P}}\right)$ and $\frac{d^{2} \pi_{m k}^{\mathcal{P}+}\left(\underline{y}_{k}^{\mathcal{P}}\right)}{d\left(\underline{y}_{k}^{\mathcal{P}}\right)}=-\left(e_{k}+h_{m k}-\alpha w_{k+1}\right) f_{k}\left(\underline{y}_{k}^{\mathcal{P}}\right)<0$. Thus, we conclude that there is an optimal value for the decision variable $\underline{y}_{k}^{\mathcal{P}}$ to maximize $\pi_{m k}^{\mathcal{P}+}\left(\underline{y}_{k}^{\mathcal{P}}\right)$. Let $\frac{d \pi_{m k}^{\mathcal{P}+}\left(\underline{y}_{k}^{\mathcal{P}}\right)}{d y_{k}^{\mathcal{P}}}=0$, we can derive the above result.

Proof of Lemma 5.9. The proof process of Lemma 5.5 is similar with that of Lemma 4.1 and thus is omitted.

Proof of Proposition 5.10. In the supplier-led model, for period $k=1, \ldots, n$, the optimal solution for the decision variables $\bar{y}_{k}^{\mathcal{R}}$ and $\underline{y}_{k}^{\mathcal{R}}$ are given by $\bar{y}_{k}^{\mathcal{R} *}=\left\{\begin{array}{ll}S_{k}^{\mathcal{R} *} & \text { if } L_{m k}^{\mathcal{R}} \leq S_{k}^{\mathcal{R} *} \\ L_{m k}^{\mathcal{R}} & \text { if } L_{m k}^{\mathcal{R}}>S_{k}^{\mathcal{R} *}\end{array}\right.$ and $y_{k}^{\mathcal{R} *}=\left\{\begin{array}{ll}s_{k}^{\mathcal{R} *} & \text { if } L_{m k}^{\mathcal{R}} \leq s_{k}^{\mathcal{R} *} \\ L_{m k}^{\mathcal{R}} & \text { if } L_{m k}^{\mathcal{R}}>s_{k}^{\mathcal{R} *}\end{array}\right.$, respectively, where $\quad S_{k}^{\mathcal{R} *}=\arg _{\max _{\bar{y}_{k}^{\mathcal{R}}}}\left\{\pi_{m k}^{\mathcal{R}+}\left(\bar{y}_{k}^{\mathcal{R}}, \underline{y}_{k}^{\mathcal{R}}\right)+\alpha E V_{m k+1}^{\mathcal{R}+}\left[\left(\underline{y}_{k}^{\mathcal{R}}-D_{k}\right)^{+}\right]\right\} \quad$ and $s_{k}^{\mathcal{R} *}=\arg \max _{\underline{y}_{k}^{\mathcal{R}}}\left\{\pi_{m k}^{\mathcal{R}+}\left(\bar{y}_{k}^{\mathcal{R}}, \underline{y}_{k}^{\mathcal{R}}\right)+\alpha E V_{m k+1}^{\mathcal{R}+}\left[\left(\underline{y}_{k}^{\mathcal{R}}-D_{k}\right)^{+}\right]\right\}$. Since $q_{w k}^{\mathcal{R} *}=\bar{y}_{k}^{\mathcal{R} *}-L_{m k}^{\mathcal{R}}$ and $q_{m k}^{\mathcal{R} *}=\bar{y}_{k}^{\mathcal{R} *}-\underline{y}_{k}^{\mathcal{R} *}$, we can derive the above result. 
Proof of Corollary 5.11. Let $U_{m k}^{\mathcal{R}+}\left(\bar{y}_{k}^{\mathcal{R}}, \underline{y}_{k}^{\mathcal{R}}\right)=\pi_{m k}^{\mathcal{R}+}\left(\bar{y}_{k}^{\mathcal{R}}, \underline{y}_{k}^{\mathcal{R}}\right)+\alpha E V_{m k+1}^{\mathcal{R}+}\left[\left(\underline{y}_{k}^{\mathcal{R}}-D_{k}\right)^{+}\right]$. It is easy to see that $\frac{\partial^{2} U_{m k}^{\mathcal{R}+}\left(\bar{y}_{k}^{\mathcal{R}}, \underline{y}_{k}^{\mathcal{R}}\right)}{\partial \bar{y}_{k}^{\mathcal{R}} \partial w_{k}}<0, \frac{\partial^{2} U_{m k}^{\mathcal{R}+}\left(\bar{y}_{k}^{\mathcal{R}}, \underline{\underline{R}}_{k}^{\mathcal{R}}\right)}{\partial \bar{y}_{k}^{\mathcal{R}} \partial o_{k}}<0$ and $\frac{\partial^{2} U_{m k}^{\mathcal{R}+}\left(\bar{y}_{k}^{\mathcal{R}}, \underline{y}_{k}^{\mathcal{R}}\right)}{\partial \bar{y}_{k}^{\mathcal{R}} \partial e_{k}}>0$, which means that $S_{k}^{\mathcal{R} *}$ is a decreasing function of $w_{k}$ and $o_{k}$, and is an increasing function of $e_{k}$. Similarly, we have $\frac{\partial^{2} U_{m k}^{\mathcal{R}}\left(\bar{y}_{k}^{\mathcal{R}}, \underline{y}_{k}^{\mathcal{R}}\right)}{\partial \underline{y}_{k}^{\mathcal{R}} \partial w_{k}}=0, \frac{\partial^{2} U_{m k}^{\mathcal{R}}+\left(\bar{y}_{k}^{\mathcal{R}}, \underline{y}_{k}^{\mathcal{R}}\right)}{\partial \underline{y}_{k}^{\mathcal{R}} \partial o_{k}}>0$ and $\frac{\partial^{2} U_{m k}^{\mathcal{R}+}\left(\bar{y}_{k}^{\mathcal{R}}, \underline{y}_{k}^{\mathcal{R}}\right)}{\partial \underline{y}_{k}^{\mathcal{R}} \partial e_{k}}<0$, which means that $s_{k}^{\mathcal{R} *}$ is a constant function of $w_{k}$, is an increasing function of $o_{k}$, and is a decreasing function of $e_{k}$.

Proof of Proposition 5.12. From equation (5.15), we have $\frac{\partial \pi_{m k}^{\mathcal{R}+}\left(\bar{y}_{k}^{\mathcal{R}}, \underline{y}_{k}^{\mathcal{R}}\right)}{\partial \bar{y}_{k}^{\mathcal{R}}}=\left(r_{k}-w_{k}-o_{k}\right)-\left(r_{k}-e_{k}\right) F\left(\bar{y}_{k}^{\mathcal{R}}\right)$, $\frac{\partial \pi_{m k}^{\mathcal{R}}\left(\bar{y}_{k}^{\mathcal{R}}, \underline{y}_{k}^{\mathcal{R}}\right)}{\partial \underline{y}_{k}^{\mathcal{R}}}=o_{k}-\left(e_{k}+h_{m k}-\alpha w_{k+1}\right) F\left(\underline{y}_{k}^{\mathcal{R}}\right), \frac{\partial^{2} \pi_{m k}^{\mathcal{R}}\left(\bar{y}_{k}^{\mathcal{R}}, \underline{y}_{k}^{\mathcal{R}}\right)}{\partial\left(\bar{y}_{k}^{\mathcal{R}}\right)^{2}}=-\left(g_{k}-e_{k}\right) f\left(\bar{y}_{k}^{\mathcal{R}}\right)<0$ and $\frac{\partial^{2} \pi_{m k}^{\mathcal{R}}\left(\bar{y}_{k}^{\mathcal{R}}, \underline{y}_{k}^{\mathcal{R}}\right)}{\partial\left(\underline{y}_{k}^{\mathcal{R}}\right)^{2}}=-\left(e_{k}+h_{m k}-\alpha w_{k+1}\right) f\left(\underline{y}_{k}^{\mathcal{R}}\right)<0$. Because $\frac{\partial^{2} \pi_{m k}^{\mathcal{R}+}\left(\bar{y}_{k}^{\mathcal{R}}, \underline{y}_{k}^{\mathcal{R}}\right)}{\partial \bar{y}_{k}^{\mathcal{R}} \partial \underline{y}_{k}^{\mathcal{R}}}=\frac{\partial^{2} \pi_{m k}^{\mathcal{R}}+\left(\bar{y}_{k}^{\mathcal{R}}, \underline{y}_{k}^{\mathcal{R}}\right)}{\partial \underline{y}_{k}^{\mathcal{R}} \partial \bar{y}_{k}^{\mathcal{R}}}=0$, we have $\left|\begin{array}{cc}\frac{\partial^{2} \pi_{m k}^{\mathcal{R}+}\left(\bar{y}_{k}^{\mathcal{R}}, \underline{y}_{k}^{\mathcal{R}}\right)}{\partial\left(\bar{y}_{k}^{\mathcal{R}}\right)^{2}} & \frac{\partial^{2} \pi_{m k}^{\mathcal{R}+}\left(\bar{y}_{k}^{\mathcal{R}}, \underline{y}_{k}^{\mathcal{R}}\right)}{\partial \bar{y}_{k}^{\mathcal{R}} \partial \underline{y}_{k}^{\mathcal{R}}} \\ \frac{\partial^{2} \pi_{m k}^{\mathcal{R}+}\left(\bar{y}_{k}^{\mathcal{R}}, \underline{y}_{k}^{\mathcal{R}}\right)}{\partial \underline{y}_{k}^{\mathcal{R}} \partial \bar{y}_{k}^{\mathcal{R}}} & \frac{\partial^{2} \pi_{m k}^{\mathcal{R}+}\left(\bar{y}_{k}^{\mathcal{R}}, \underline{y}_{k}^{\mathcal{R}}\right)}{\partial\left(\underline{y}_{k}^{\mathcal{R}}\right)^{2}}\end{array}\right|>$

variables $\bar{y}_{k}^{\mathcal{R}}$ and $\underline{y}_{k}^{\mathcal{R}}$ to maximize $\pi_{m k}^{\mathcal{R}+}\left(\bar{y}_{k}^{\mathcal{R}}, \underline{y}_{k}^{\mathcal{R}}\right)$. Let $\frac{\partial \pi_{m k}^{\mathcal{R}+}\left(\bar{y}_{k}^{\mathcal{R}}, \underline{y}_{k}^{\mathcal{R}}\right)}{\partial \bar{y}_{k}^{\mathcal{R}}}=0$ and $\frac{\partial \pi_{m k}^{\mathcal{R}+}\left(\bar{y}_{k}^{\mathcal{R}}, \underline{y}_{k}^{\mathcal{R}}\right)}{\partial \underline{y}_{k}^{\mathcal{R}}}=0$, we can derive the above result.

Proof of Lemma 7.1. The proof process of Lemma 7.1 is similar with that of Lemma 4.1 and thus is omitted.

Proof of Proposition 7.2. In the integrated model, for period $k=1, \ldots, n$, the optimal solution for the decision variable $y_{c k}^{\mathcal{J}}$ is characterized by $y_{c k}^{\mathcal{J} *}=\left\{\begin{array}{ll}S_{k}^{\mathcal{J} *} & \text { if } L_{c k}^{\mathcal{J}} \leq S_{k}^{\mathcal{J} *}, \\ L_{c k}^{\mathcal{J}} & \text { if } L_{c k}^{\mathcal{J}}>S_{k}^{\mathcal{J} *},\end{array}\right.$ where $S_{k}^{C *}=\arg \max _{\bar{y}_{k}^{\mathcal{J}}}\left\{\pi_{c k}^{\mathcal{J}+}\left(\bar{y}_{k}^{\mathcal{J}}\right)+\alpha E V_{c k+1}^{\mathcal{J}+}\left[\left(\bar{y}_{k}^{\mathcal{J}}-D_{k}\right)^{+}\right]\right\}$. Given that $q_{s k}^{\mathcal{J} *}=\bar{y}_{k}^{\mathcal{J} *}-L_{c k}^{\mathcal{J}}$, we can derive the above result.

Proof of Corollary 7.3. Let $U_{c k}^{\mathcal{J}+}\left(\bar{y}_{k}^{\mathcal{J}}\right)=\pi_{c k}^{\mathcal{J}+}\left(\bar{y}_{k}^{\mathcal{J}}\right)+\alpha E V_{c k+1}^{\mathcal{J}+}\left[\left(\bar{y}_{k}^{\mathcal{J}}-D_{k}\right)^{+}\right]$. It is easy to see that $\frac{\partial^{2} U_{c k}^{\mathcal{J}+}\left(\bar{y}_{k}^{\mathcal{J}}\right)}{\partial \bar{y}_{k}^{\mathcal{J}} \partial c_{k}}<0$, which means that $S_{k}^{\mathcal{J} *}$ is a decreasing function of $c_{k}$.

Proof of Proposition 7.4. From equation (7.5), we derive $\frac{d \pi_{c k}^{\mathcal{J}+}\left(\bar{y}_{k}^{\mathcal{J}}\right)}{d y_{k}^{\mathcal{J}}}=\left(r_{k}-c_{k}\right)-\left(r_{k}+h_{s k}-\alpha c_{k+1}\right) F_{k}\left(\bar{y}_{k}^{\mathcal{J}}\right)$ and $\frac{d^{2} \pi_{c k}^{\mathcal{J}+}\left(\bar{y}_{k}^{\mathcal{J}}\right)}{d\left(\bar{y}_{k}^{\mathcal{J}}\right)^{2}}=-\left(r_{k}+h_{s k}-\alpha c_{k+1}\right) f_{k}\left(\bar{y}_{k}^{\mathcal{J}}\right)<0$. Thus, we conclude that there is an optimal value for the decision variable $\bar{y}_{k}^{\mathcal{J}}$ to maximize $\pi_{c k}^{\mathcal{J}+}\left(\bar{y}_{k}^{\mathcal{J}}\right)$. Let $\frac{d \pi_{c k}^{\mathcal{J}+}\left(\bar{y}_{k}^{\mathcal{J}}\right)}{d y_{k}^{\mathcal{J}}}=0$, we can derive the above result.

Proof of Proposition 7.5. In the manufacturer-led supply chain, the wholesale price and put option portfolio contract that satisfies $\frac{w_{k}+o_{k}-c_{k}}{e_{k}+h_{s k}-\alpha c_{k+1}}=\frac{r_{k}-c_{k}}{r_{k}+h_{s k}-\alpha c_{k+1}}$ can provide the supplier with an incentive to produce nearly the same quantity of the components as the integrated supply chain. With some algebra we derive the above result.

Proof of Proposition 7.6. If the manufacturer-led supply chain is coordinated bilaterally, we have $\frac{d \pi_{m k}^{\mathcal{P}+}\left(\underline{\mathcal{P}}_{k}^{\mathcal{P}}\right)}{d y_{k}^{\mathcal{P}}}=o_{k}-\frac{\left(w_{k}+o_{k}-c_{k}\right)\left(r_{k}+h_{s k}-\alpha c_{k+1}\right)-\left(r_{k}-c_{k}\right)\left[\left(h_{s k}-\alpha c_{k+1}\right)-\left(h_{m k}-\alpha w_{k+1}\right)\right]}{r_{k}-c_{k}} F_{k}\left(\underline{y}_{k}^{\mathcal{P}}\right)$. If $o_{k}<\frac{\left(r_{k}-c_{k}\right)\left[\left(h_{s k}-\alpha c_{k+1}\right)-\left(h_{m k}-\alpha w_{k+1}\right)\right]-\left(w_{k}-c_{k}\right)\left(r_{k}+h_{s k}-\alpha c_{k+1}\right)}{c_{k}+h_{s k}-\alpha c_{k+1}}$, we have $s_{k}^{\mathcal{P}+}=0$. 
If $\frac{\left(r_{k}-c_{k}\right)\left[\left(h_{s k}-\alpha c_{k+1}\right)-\left(h_{m k}-\alpha w_{k+1}\right)\right]-\left(w_{k}-c_{k}\right)\left(r_{k}+h_{s k}-\alpha c_{k+1}\right)}{c_{k}+h_{s k}-\alpha c_{k+1}}<o_{k}<\frac{\left(w_{k}-c_{k}\right)\left(e_{k}+h_{m k}-\alpha w_{k+1}\right)}{\left(h_{s k}-\alpha c_{k+1}\right)-\left(h_{m k}-\alpha w_{k+1}\right)}$, we have $s_{k}^{\mathcal{P}+}=F_{k}^{-1}\left(\frac{o_{k}\left(r_{k}-c_{k}\right)}{\left(w_{k}+o_{k}-c_{k}\right)\left(r_{k}+h_{s k}-\alpha c_{k+1}\right)-\left(r_{k}-c_{k}\right)\left[\left(h_{s k}-\alpha c_{k+1}\right)-\left(h_{m k}-\alpha w_{k+1}\right)\right]}\right)$.

Proof of Proposition 7.7. In the supplier-led supply chain, the wholesale price and put option portfolio contract that satisfies $\frac{r_{k}-w_{k}-o_{k}}{r_{k}-e_{k}}=\frac{r_{k}-c_{k}}{r_{k}+h_{s k}-\alpha c_{k+1}}$ can provide the manufacturer with an incentive to buy nearly the same quantity of components as the production quantity of the integrated supply chain system. With some algebra we derive the above result.

Acknowledgements. The paper is partially supported by National Natural Science Foundation of China (71802168), Humanities and Social Science Fund of Ministry of Education of China (18YJC630165, 20YJC630020) and Fund of Sichuan Information Management and Service Research Center (SCXX2018ZD05).

\section{REFERENCES}

[1] H.V. Arani, M. Rabbani and H. Rafiei, A revenue-sharing option contract toward coordination of supply chains. Int. J. Prod. Econ. 178 (2016) $42-56$.

[2] P. Basu, Q. Liu and J. Stallaert, Supply chain management using put option contracts with information asymmetry. Int. J. Prod. Res. 57 (2019) 1772-1796.

[3] S. Benjaafar, D. Chen and Y. Yu, Optimal policies for inventory systems with concave ordering costs. Nav. Res. Logist. 65 (2018) 291-302.

[4] M. Bhattacharyya and S.S. Sana, A mathematical model on eco-friendly manufacturing system under probabilistic demand. RAIRO:OR 53 (2019) 1899-1913

[5] F. Chen and M. Parlar, Value of a put option to the risk-averse newsvendor. IIE Trans. 39 (2007) 481-500.

[6] L.G. Chen, D. Ding and J. Ou, Power structure and profitability in assembly supply chains. Prod. Oper. Manage. 23 (2014) $1599-1616$.

[7] C. Chiang and G.J. Gutierrez, Optimal control policies for a periodic review inventory system with emergency orders. Nav. Res. Logist. 45 (1998) 187-204.

[8] K. Eriksson, An option mechanism to coordinate a dyadic supply chain bilaterally in a multi-period setting. Omega 88 (2019) 196-209.

[9] E.A. Feinberg and Y. Liang, Structure of optimal policies to periodic-review inventory models with convex costs and backorders for all values of discount factors. Ann. Oper. Res. (2017). DOI: 10.1007/s10479-017-2548-6.

[10] B.C. Giri and S. Sharma, Optimal production policy for a closed-loop hybrid system with uncertain demand and return under supply disruption. J. Clean. Prod. 112 (2016) 2015-2028.

[11] C.C. Hsieh, Y.L. Chang and C.H. Wu, Competitive pricing and ordering decisions in a multiple-channel supply chain. Int. J. Prod. Econ. 54 (2014) 156-165.

[12] Z. Hu, J. Tian and G. Feng, A relief supplies purchasing model based on a put option contract. Comput. Ind. Eng. 127 (2019) $253-262$.

[13] O. Jadidi, M.Y. Jaber and S. Zolfaghari, Joint pricing and inventory problem with price dependent stochastic demand and price discounts. Comput. Ind. Eng. 114 (2017) 45-53.

[14] X. Li, Y. Li and X. Cai, On a multi-period supply chain system with supplementary order opportunity. Eur. J. Oper. Res. 209 (2011) 273-284.

[15] Y. Lu and M. Song, Inventory control with a fixed cost and a piecewise linear convex cost. Prod. Oper. Manage. 23 (2014) $1966-1984$.

[16] Y. Lu, M. Song and Y. Yang, Joint inventory and pricing coordination with incomplete demand information. Prod. Oper. Manage. 25 (2016) 701-718.

[17] N.M. Modak, Exploring Omni-channel supply chain under price and delivery time sensitive stochastic demand. Supply Chain Forum: Int. J. 18 (2017) 218-230.

[18] N.M. Modak and P. Kelle, Managing a dual-channel supply chain under price and delivery-time dependent stochastic demand. Eur. J. Oper. Res. 272 (2019) 147-161.

[19] N.M. Modak and P. Kelle, Using social work donation as a tool of corporate social responsibility in a closed-loop supply chain considering carbon emissions tax and demand uncertainty. J. Oper. Res. Soc. (2019). DOI: 10.1080/01605682.2019.1654413

[20] S.S. Sana, Price sensitive demand with random sales price - a newsboy problem. Int. J. Syst. Sci. 43 (2012) $491-498$.

[21] R. Shi, J. Zhang and J. Ru, Impacts of power structure on supply chains with uncertain demand. Prod. Oper. Manage. 22 (2013) 1232-1249.

[22] H. Song, L. Ran and J. Shang, Multi-period optimization with loss-averse customer behavior: joint pricing and inventory decisions with stochastic demand. Expert Syst. App. 72 (2017) 421-429.

[23] N. Wan and X. Chen, The role of put option contracts in supply chain management under inflation. Int. Trans. Oper. Res. 26 (2019) 1451-1474 
[24] C. Wang and X. Chen, Joint order and pricing decisions for fresh produce with put option contracts. J. Oper. Res. Soc. 69 (2018) 474-484.

[25] L. Wang and Q. Song, Pricing policies for dual-channel supply chain with green investment and sales effort under uncertain demand. Math. Comput. Simul. 171 (2020) 79-93.

[26] W. Xue, O.C. Demirag and B. Niu, Supply chain performance and consumer surplus under alternative structures of channel dominance. Eur. J. Oper. Res. 239 (2014) 130-145.

[27] W. Xue, L. Ma and H. Shen, Optimal inventory and hedging decisions with CVaR consideration. Int. J. Prod. Econ. 162 (2015) 70-82.

[28] L. Yang, G. Cai and J. Chen, Push, pull, and supply chain risk-averse attitude. Prod. Oper. Manage. 27 (2018) $1534-1552$.

[29] J.L. Zhang, J. Chen and C.Y. Lee, Coordinated pricing and inventory control problems with capacity constraints and fixed ordering cost. Nav. Res. Logist. 59 (2012) 376-383. 